\title{
Modeling and Characterization of the Uplink and Downlink Exposure in Wireless Networks
}

\author{
Anis Krayni, ${ }^{1,2}$ Abdelhamid Hadjem, ${ }^{2}$ Günter Vermeeren, ${ }^{3}$ Alain Sibille, \\ Christophe Roblin, ${ }^{1}$ Wout Joseph, ${ }^{3}$ Luc Martens, ${ }^{3}$ and Joe Wiart ${ }^{1}$ \\ ${ }^{1}$ Telecom ParisTech, Paris, France \\ ${ }^{2}$ Whist Lab Common Laboratory of Orange Labs and Institut Telecom, Paris, France \\ ${ }^{3}$ Ghent University, Ghent, Belgium \\ Correspondence should be addressed to Anis Krayni; anis.krayni@gmail.com
}

Received 4 January 2017; Revised 14 April 2017; Accepted 3 May 2017; Published 11 June 2017

Academic Editor: Antonio Iodice

Copyright (C) 2017 Anis Krayni et al. This is an open access article distributed under the Creative Commons Attribution License, which permits unrestricted use, distribution, and reproduction in any medium, provided the original work is properly cited.

\begin{abstract}
This paper deals with a new methodology to assess the exposure induced by both uplink and downlink of a cellular network using 3D electromagnetic simulations. It aims to analyze together the exposure induced by a personal device (uplink exposure) and that induced by a base station (downlink exposure). The study involved the major parameters contributing to variability and uncertainty in exposure assessment, such as the user's posture, the type of wireless device, and the propagation environment. Our approach is relying basically on the modeling of the power radiated by the personal device and the ambient electric field, while taking into account the effects of human body shadowing and the propagation channel fluctuations. The exposure assessment as well as the human-wave interactions has been simulated using the finite difference in time domain method (FDTD). In uplink scenarios, four FDTD simulations were performed with a child model, used in two postures (sitting and standing) and in two usage scenarios (voice and data), which aimed to examine the exposure induced by a mobile phone and a tablet emitting, respectively, at $900 \mathrm{MHz}$ and $1940 \mathrm{MHz}$. In the downlink scenario, a series of FDTD simulations of an exposure to a single plane wave and multiplane waves have been conducted, and an efficient metamodeling of the exposure using the Polynomial Chaos approach has been developed.
\end{abstract}

\section{Introduction}

The rapid developments in wireless network technology have strengthened the presence of electromagnetic waves in our everyday lives. Hence, the exposure to near and far electromagnetic fields is becoming increasingly a matter of public concern. The exposure is determined by the SAR (Specific Absorption Rate) (W/Kg), which quantifies the power absorbed by human tissues from electromagnetic radiations. Several studies have been conducted to characterize, on one hand, the exposure induced by the base stations (downlink exposure) [1] and, on the other hand, that induced by wireless devices (uplink exposure) [2]. In most of these studies, both types of exposure were studied separately. However, in the context of wireless networks that are now so widely present in everyone's environment, a reliable characterization of exposure requires taking into account both uplink and downlink radio waves. Basically, for a given duplex communication, these waves are not independent and involve a power management protocol [3]. The downlink radiation is mainly impacted by the propagation environment, particularly through the attenuation suffered by the signal wave before arriving at the receiver [4]. In the other side, other than the propagation environment, the uplink radiation is dependent on the user's activity, on the network (e.g., the rate and the QoS), and fundamentally on the performance of the device's antenna [5]. Actually, the wireless devices are often placed in the proximity of the user's body, which is a conducting system. Accordingly, a strong coupling effect takes place between the human tissues and the antenna [6]. These interactions can affect severely the antenna radiation properties, which are strongly involved in adjusting the uplink power [7]. The purpose of this paper is to discuss a numerical approach to characterize the variability associated 
with both links radiations as well as the resulting exposure, using electromagnetic simulations and statistical analysis.

Compared to previous research $[8,9]$, this work involves the use of an anatomic child model under various postures, including standing and sitting position. Furthermore, it aims at introducing a methodology for characterizing the uplink power fluctuations as a function of shadowing effects caused by the human body and the multipath fading. In the downlink, this study deals with a metamodeling approach allowing prediction analytically of the exposure induced by a complex propagation environment.

In fact, a FDTD simulation incorporating a human body with a resolution of 1-2 $\mathrm{mm}$ is very expensive in time calculation and resources, which often impedes the study of a large number of exposure configurations. Our proposed method consists of extracting an input-output transfer function, linking the exposure induced by multiple plane waves to the parameters contributing in the variability of their total electric field, using a regression metamodel approach in the postprocessing of a finite subset of FDTD simulations.

This study is a part of an European project called LEXNET [10], supported by the European Commission under the FP7, was established to minimize the exposure induced by wireless systems. The rest of this paper is organized as follows. The second section presents the methodology used in the modeling of the uplink power and the electric field received from a fixed base station. The third section illustrates the materials used to prepare the simulations. Section 4 is reserved to discuss the results as well as the statistical analysis of the uplink exposure variability. Section 5 focuses on the investigation of the exposure to single and multiple plane waves. In this section, we discuss the use of Polynomial Chaos (PC) [11] approach in the metamodeling of the multipath exposure. In the last section, we apply the proposed results to realistic traffic measurements. A global conclusion is drawn in the end of the paper.

\section{Exposure Assessment}

In wireless systems, the total exposure induced by such a radio communication is given as the sum of the uplink exposure and the downlink exposure. Two quantities are often used to characterize such exposure: the local SAR and the global SAR. The first is defined as the power absorbed over a cube of $10 \mathrm{~g}$, whose peak value is denoted by $\mathrm{SAR}_{10 \mathrm{~g}}^{\text {peak }}$. The second is the whole-body average $S A R_{W B}$, well known as the ratio of the absorbed power to the whole-body weight.

During a radio communication, the global induced exposure can be deduced from the following system of equations:

SAR:

$$
\begin{aligned}
& \operatorname{SAR}_{10 \mathrm{~g}}^{\text {peak }}=\max \{\Lambda\} \\
& \qquad \text { where } \Lambda=P_{r} \mathrm{SAR}_{10 \mathrm{~g}}^{\mathrm{UL} *}+E_{a}^{2} \mathrm{SAR}_{10 \mathrm{~g}}^{\mathrm{DL} *} \\
& \mathrm{SAR}_{\mathrm{WB}}=P_{\mathrm{in}} \mathrm{SAR}_{\mathrm{WB}}^{\mathrm{UL} *}+E_{a}^{2} \mathrm{SAR}_{\mathrm{WB}}^{\mathrm{DL} *},
\end{aligned}
$$

where $S A R_{10 \mathrm{~g}}^{\mathrm{UL} *}$ and $S A R_{\mathrm{WB}}^{\mathrm{UL} *}$ are, respectively, the $\mathrm{SAR}_{10 \mathrm{~g}}^{\text {peak }}$ and $S A R_{W B}$ induced by a fixed uplink power of $1 \mathrm{~W}$, respectively. $\mathrm{SAR}_{10 \mathrm{~g}}^{\mathrm{DL} *}$ and $\mathrm{SAR}_{\mathrm{WB}}^{\mathrm{DL} *}$ are, respectively, the $\mathrm{SAR}_{10 \mathrm{~g}}$ matrix and $S A R_{W B}$ induced by an ambient electric field of $1 \mathrm{~V} / \mathrm{m}$, respectively. It should be mentioned that the $S A R_{10 \mathrm{~g}}$ matrix is used to regroup all $S A R_{10 g}$, those calculated over the whole body, in a $3 \mathrm{D}$ matrix whose dimensions are equal to the dimensions of the user's body $[12,13] . P_{\text {in }}(\mathrm{W})$ and $E_{a}(\mathrm{~V} / \mathrm{m})$ represent the input power delivered to the personal device and the ambient electric field, respectively.

In both above relationships, the exposure level is calculated using the proportional relationship between the absorbed power and the electromagnetic radiation, which is considered as an unknown time-varying parameter.

2.1. Power Radiated by a Personal Device. This part is devoted to the modeling of the input power delivered to the personal device during an uplink communication. Obviously, this quantity depends on various parameters, including the antenna losses, the propagation channel (shadowing effects, multipath fluctuations), and the network's requirements in terms of the signal to-noise-ratio (SNR), especially when using a power management protocol [14]. Several studies have been performed to model the uplink power on a wireless network [15]. The vast majority of these studies were largely based on the use of statistical models for multipath channel to characterize the power fluctuations in different propagation scenarios. Among the famous models, we can mention the WINNER II project [16], which provides the main propagation characteristics of a set of specific environments, such as rural, urban, and semiurban regions. For each environment, it gives a prediction of the number of paths existing between any system "transmitter-receiver" as well as their amplitudes, phases, and arrivals and departures directions. In a typical multipath propagation channel, the $P_{\text {in }}$ power should satisfy the following equation:

$$
P_{\text {in }} \sum_{n=0}^{N_{p}} a_{n}^{2}\left(\tau_{n}\right) G_{e}\left(\phi_{n}, \theta_{n}\right)=\alpha,
$$

where $\alpha$ is the power radiated by the personal device, which takes into account the user-induced losses and the fluctuations of the radio link. Otherwise, $P_{\text {in }}$ is the antenna input power. This unknown quantity is given as the sum of the power absorbed by the users body and the useful power, which can be used to ensure the radio communication. The consideration of this basic relation is justified by the fact that the antenna is assumed well matched (without losses).

To focus only on the impact of the propagation environment and antenna performances, without taking into account the network requirements, the power $\alpha$ is kept constant and equal to $1 \mathrm{~mW}$. $G_{e}$ is the effective gain, which is the gain of the couple "user-antenna." This couple is considered invariant. In fact, the position of the device with respect to the user's body is assumed fixed during this study. $N_{p}$ denotes the total number of paths, including the direct path Line-ofSight (LOS) and non-line-of-sight (NLOS) path. Each path is parametrized by two angles: an azimuth angle $\phi_{n}$ and 
an elevation angle $\theta_{n}$. These angles are assumed to follow a discrete uniform distribution between $0^{\circ}$ and $360^{\circ}$ and a normal distribution with a mean of $0^{\circ}$ and a standard deviation of $0^{\circ}$, respectively. The elevation $0^{\circ}$ is associated with the horizontal plane.

To model the losses induced by the distance, we have intervened an attenuation coefficient $a_{n}$. This parameter is generated using an exponential function that depends on the delay spread $\tau_{n}(s)$.

$$
a_{n}^{2}\left(\tau_{n}\right) \propto e^{-\tau_{n} / \sigma_{s}},
$$

where $\tau_{n}$ represents the time delay between transmission and reception of a signal. This time parameter is assumed to be uniformly distributed and increasingly arranged between 0 and $\tau_{N} .0$ is associated with the first path, which is often the LOS. $\sigma_{s}$ is the root mean square (rms) delay spread.

Three propagation scenarios have been considered:

(i) Line-of-Sight scenario (LOS): a very typical propagation of a low probability of occurrence.

(ii) Non-Line-Of-Sight Scenario (NLOS): common scenario, such as indoor scenarios.

(iii) Combined LOS/NLOS: common scenario (e.g., urban scenarios).

In the first case, we recall that the radio transmission can take place only under the LOS path, while in the second scenario we assume the existence of only indirect paths (NLOS) between the user and the base station. The last scenario assumes the existence of both types of paths together during the communication.

To separate mappings between these different scenarios, we note that the first component in (2), $a_{0}^{2}\left(\tau_{0}\right) G_{e}\left(\phi_{0}, \theta_{0}\right)$, is associated with the LOS path. In LOS environment, $a_{0}$ takes 1 by assuming a constant path loss normalized to be one.

In pure NLOS scenario, $a_{0}$ is chosen equal to 0 , while in mixed LOS/NLOS scenario $a_{0}$ is subject to the Rice factor $(K)$, which is given as follows:

$$
K=\frac{a_{0}^{2}\left(\tau_{0}\right)}{\sum_{n=1}^{N_{p}} a_{n}^{2}\left(\tau_{n}\right)} .
$$

All attenuation coefficients $a_{k \geq 0}$ are normalized so that the total sum is equal to one.

The main characteristics of each propagation scenario are illustrated in Table 1. A detailed explanation of the proposed methodology as well as considered assumptions is offered in [17].

2.2. The Ambient Electric Field. Turning to the downlink radiations, throughout this study we assume that the electric field emitted by the base station is considered as a uniform plane wave. Similarly to the uplink case, the ambient electric field can be composed of single or multiple plane waves arriving form random directions, with various propagation

\begin{tabular}{|c|c|c|c|}
\hline & LOS & NLOS & LOS/NLOS \\
\hline$\theta_{n}$ & & & \\
\hline Gaussian & $\mathrm{N}(0,30)$ & $\mathrm{N}(0,30)$ & $\mathrm{N}(0,30)$ \\
\hline$\phi_{n}$ & & & \\
\hline${ }_{K}$ Uniform & $\mathrm{U}[0,360]$ & $\mathrm{U}[0,360]$ & $\mathrm{U}[0,360]$ \\
\hline Log-normal & & & $\operatorname{LN}(6,7)$ \\
\hline$N_{p}$ & & $\mathrm{~N}(6,3.5)$ & $\mathrm{N}(10,6)$ \\
\hline$a_{k}$ & & & \\
\hline$\sigma_{s}(\mathrm{~ns})$ & & 55 & 22 \\
\hline
\end{tabular}

TABLE 1: Propagation environment parameters.

characteristics. Briefly, in a given location $r$, the total ambient field $\vec{E}_{a}$ can be expressed as the following sum:

$$
\overrightarrow{E_{a}}=\sum_{n=1}^{N_{p}}{\overrightarrow{E_{n}}}^{(\mathrm{V})}\left(\alpha_{n}, \phi_{n}, \theta_{n}\right)+{\overrightarrow{E_{n}}}^{(\mathrm{H})}\left(\alpha_{n}, \phi_{n}, \theta_{n}\right),
$$

where $N_{p}$ is the number of waves. ${\overrightarrow{E_{n}}}^{(\mathrm{V})}$ and ${\overrightarrow{E_{n}}}^{(\mathrm{H})}$ are, respectively, the vertical and horizontal polarized components of the $n$th incident plane wave. $\alpha_{n}, \phi_{n}$, and $\theta_{n}$ are the phase angle, the azimuth angle of arrival, and the elevation angle of arrival, respectively.

The first two angles are assumed to follow a uniform distribution between $0^{\circ}$ and $360^{\circ}$, whereas the third angle $\left(\theta_{n}\right)$ is assumed to obey a normal distribution with a mean $\mu$ of $0^{\circ}$ and a standard deviation $\sigma$ of $20^{\circ}$.

Assuming that the original wave emitted from the base station is vertically polarized, the power received at this polarization can be described by a decreasing exponential function depending on the time parameter described above $\left(\tau_{n}, \sigma_{s}\right)$ :

$$
\left\|E_{n}^{(\mathrm{V})}\right\|^{2}=\frac{P_{0}}{N_{P}} e^{-\tau_{n} / \sigma_{s}},
$$

where $P_{0}$ is the power associated with the original wave emitted from the base station. As a result of multiple scattering and reflection, the original signal can undergo modification of its polarization. Hence, the signal arriving at the receiver is mostly composed from vertically and horizontally polarized waves. The ratio of the power received in the vertical polarization to that received in the horizontal polarization is given by the cross polarization ratio $\mathrm{XPR}_{n}$ :

$$
\mathrm{XPR}_{n}=\left\|\frac{E_{n}^{(\mathrm{V})}}{E_{n}^{(\mathrm{H})}}\right\|^{2} .
$$

This parameter is assumed to obey a log-normal distribution with a mean 0 and standard deviation 7 [18].

The squares of all vertical and horizontal polarized components are normalized to their total sum. We have always

$$
\sum_{n=1}^{N_{p}}\left\|E_{n}^{(\mathrm{V})}\right\|^{2}+\left\|E_{n}^{(\mathrm{H})}\right\|^{2}=1
$$




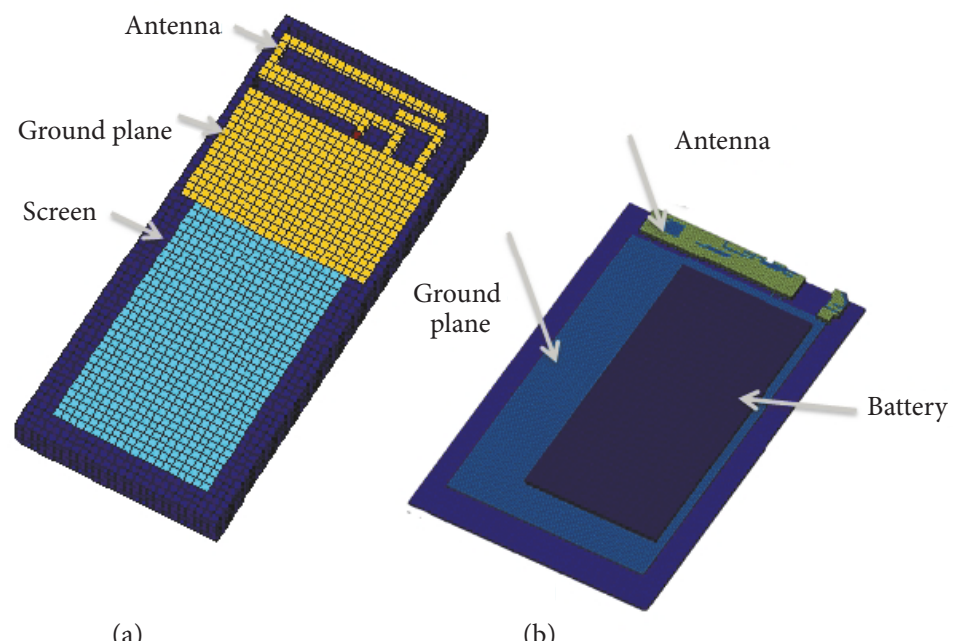

(a)

(b)

Figure 1: Wireless devices developed using MATLAB: (a) a mobile phone and (b) a tablet.

\section{Materials}

3.1. Realistic Human Phantom. To model the user body, we use an anatomically realistic human phantom Eartha, an 8year-old child selected from the Virtual Family [19]. This phantom is represented by a $3 \mathrm{D}$ heterogeneous matrix having 77 different labels, which are devoted to label the various child tissues. Using a deformation tool (EMPIRE Poser) [20], we made many elastic deformations on the standard model whose goal is to create realistic postures of the different wireless device usage, including mainly voice calling (in standing and sitting posture) and web browsing with a tablet (standing and sitting). All of these models are designed with a resolution of $2 \mathrm{~mm}$. The dielectric properties of various tissues are assigned according to the data available in [21].

Concerning the radiation sources, we developed simplified numerical models for a mobile phone and a tablet operating at $900 \mathrm{MHz}$ and $1940 \mathrm{MHz}$ (see Figure 1).

Different antennas were then validated using FDTD simulations (in free space). As regards the reflection coefficient $S_{11}$, the obtained results can be considered acceptable. As presented in Figure 2, the $S_{11}$ graph shows a significant resonance at the frequency of simulation, with a return loss below $-10 \mathrm{~dB}$ with both antennas.

3.2. Exposure Simulations. We distinguish two types of FDTD simulations: simulations with personal devices and simulations with Huygens box. To study the exposure induced by a personnel device, the developed wireless devices are positioned close to the numerical phantom depending on usage scenario, as illustrated in Figure 3. Each uplink simulation starts with injecting an electric signal in the antenna to create an electric field near to the child phantom. Then, we integrate the iterative FDTD process to compute the electric field inside the child body. In the downlink simulation, we use the Huygens box excited by a plane wave [22].

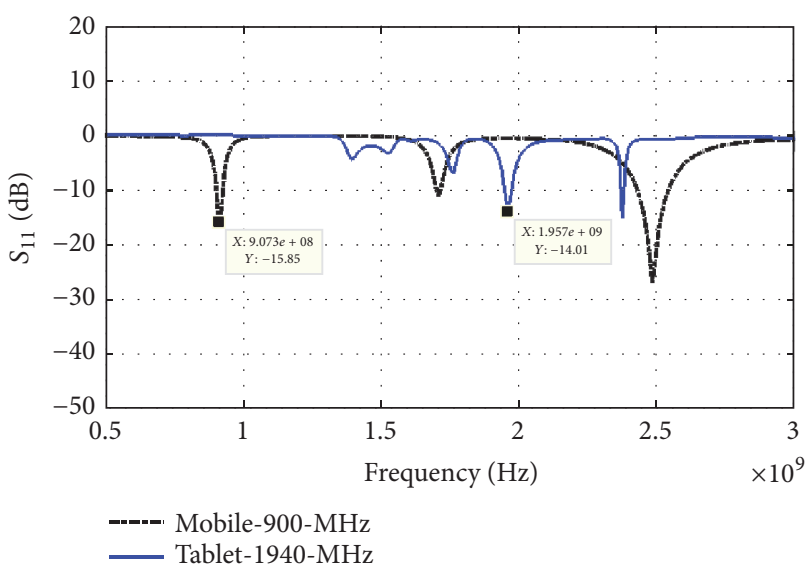

FIGURE 2: Reflection coefficient $S_{11}$ obtained from FDTD simulations in "free space."

\section{Uplink Exposure: Results and Analysis}

We begin by presenting the results obtained with the mobile phone at $900 \mathrm{MHz}$ and the tablet at $1940 \mathrm{MHz}$. Table 2 gives values of the radiation efficiency, the power absorbed by the child's body, the $\mathrm{SAR}_{\mathrm{WB}}$, and the $\mathrm{SAR}_{10 \mathrm{~g}}^{\text {peak }}$. All of these values are normalized to an input power of $1 \mathrm{~W}$. The results show that almost $90 \%$ of the input power is absorbed by the child in either sitting or standing postures. Most of the power is absorbed by the head and the left hand (that holds the mobile phone).

In the case of the tablet, the power absorbed by the child was around 35\% (standing) and 45\% (sitting). Figure 4 shows the sagittal $y z$ plane distribution of the power absorbed by the whole phantom. As shown, the level of the power absorbed reached its maximum peak in the location of the personal device.

Basically, in the case of an exposure to a personnel device, the exposure dose and especially the $\mathrm{SAR}_{10 \mathrm{~g}}^{\text {peak }}$ are very 
TABLE 2: The absorbed power and the $\mathrm{SAR}_{\mathrm{WB}}$ normalized to an input power of $1 \mathrm{~W}$.

\begin{tabular}{|c|c|c|c|c|}
\hline & Radiation efficiency (\%) & Absorbed power (\%) & $\begin{array}{l}\mathrm{SAR}_{\mathrm{WB}} \\
\mathrm{W} \cdot \mathrm{kg}^{-1}\end{array}$ & $\begin{array}{l}\mathrm{SAR}_{10 \mathrm{~g}}^{\text {peak }} \\
\mathrm{W} \cdot \mathrm{kg}^{-1}\end{array}$ \\
\hline Voice-standing & 10 & 90 & 0.029 & 3.61 \\
\hline Voice-sitting & 10 & 90 & 0.029 & 4.7 \\
\hline Tablet-standing & 65 & 35 & 0.011 & 3.67 \\
\hline Tablet-sitting & 65 & 35 & 0.014 & 4.44 \\
\hline
\end{tabular}

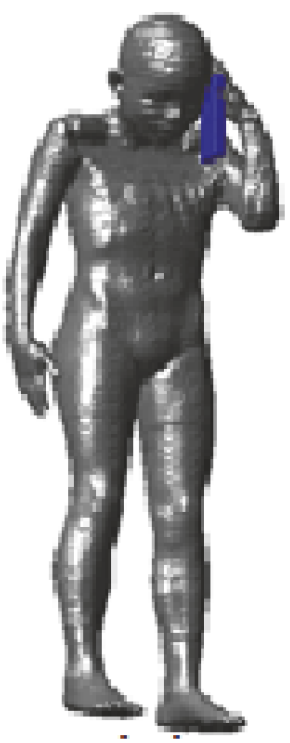

(a)

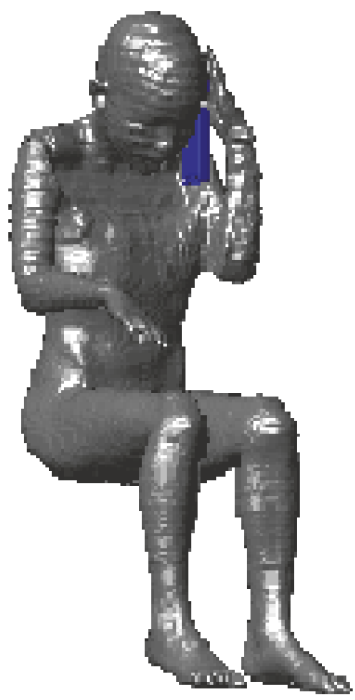

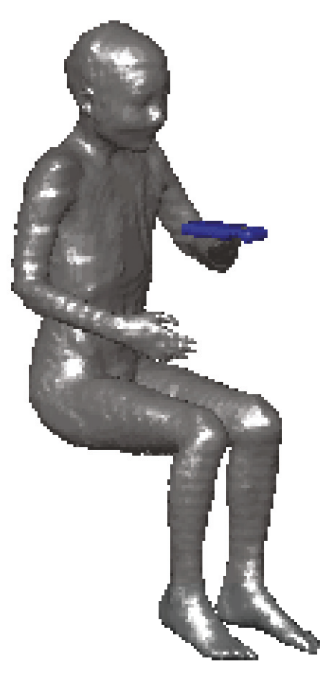

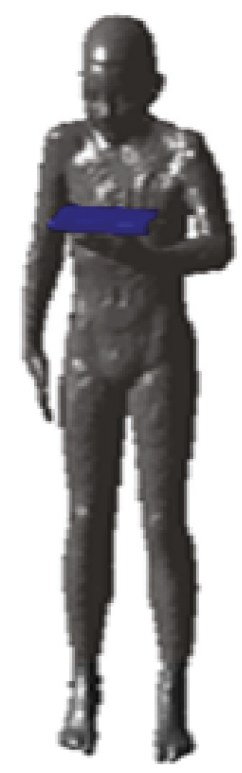

(b)

FIGURE 3: Different postures: (a) voice-standing and sitting and (b) data-standing and sitting.
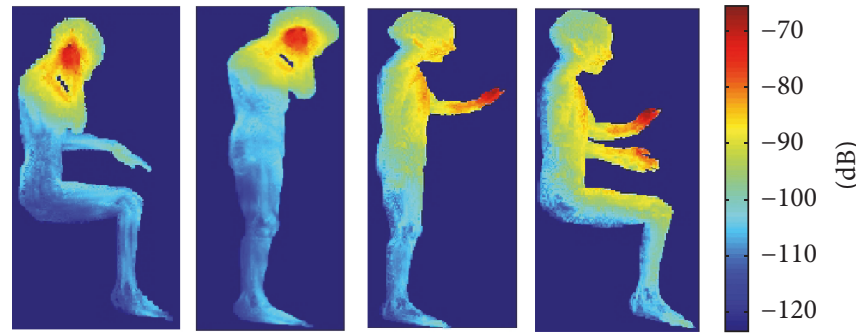

FIGURE 4: Distribution of the absorbed power $(\mathrm{db})$ inside the numerical phantom for an input power of $1 \mathrm{~W}$.

sensitive to the positioning of the antenna with respect to the user's body. For instance, a small change in the position of the mobile phone has led to a variation of $30 \%$ between both $\mathrm{SAR}_{10 \mathrm{~g}}^{\text {peak }}$ obtained with standing and sitting postures.

Turning to the converse effect, we noticed that the user's proximity caused a considerable degradation of the radiation pattern of the device's antenna. Figure 5 shows a comparison between the radiation pattern obtained with the mobile phone in the presence of the child (in a standing posture) and that calculated in free space. Briefly, we can see that a large part of the radiation pattern has been distorted by the left hand and the head, which caused the modification of the directivity of the antenna (becomes more directional in the opposite direction of the head).

Given these effects, such a characterization of the antenna gain is considered an essential step in predicting the uplink power while relying on the power fluctuation model given in the Section 2.1. For full clarity, we again stress that the variation in $T_{x}$ power stems from the power control, which is enforced by the base station according to its received power, sensitively determined by the radio channel.

A summary of the methodology is given in the diagram displayed in Figure 6. To sum up, this approach uses a FDTD simulation for characterizing the antenna's gain (in 


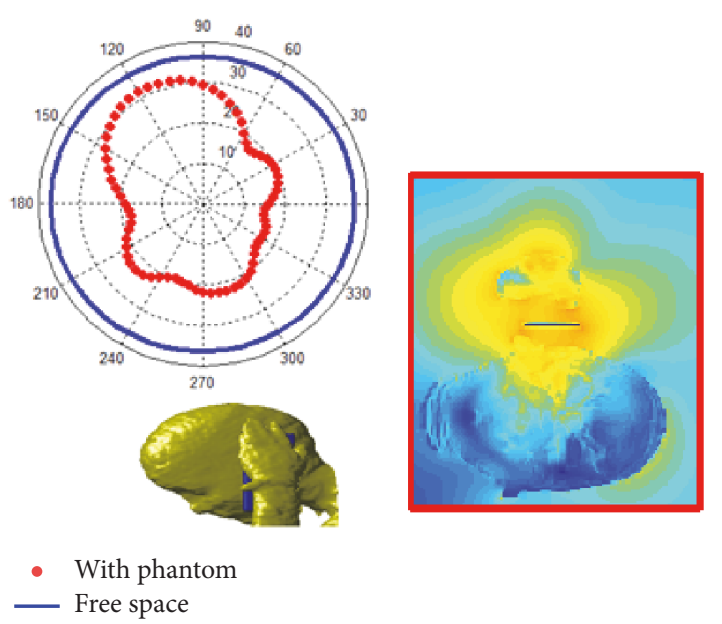

FIGURE 5: Effects of the head on the radiation pattern of the mobile phone at $900 \mathrm{MHz}$.

the presence of the user's body) as well as the induced exposure. The antenna gain obtained is then combined with a propagation model, whose aim is to analyze the variability of the input power.

To characterize the variability of the input power, a set of $10^{4}$ values has been randomly generated for each propagation scenario (LOS, NLOS/LOS, and NLOS). With the different scenarios propagation, the statistical adjustment of obtained values showed that the distribution of the input power can be fitted by a log-normal distribution, whose parameters (mean and standard deviation) depend on the propagation scenario. Due to limited space, we illustrate a few results.

As an example of results, Figure 7 shows a comparison between the log-normal distribution and the empirical distribution of a set of $10^{4}$ values of the input power obtained with the posture voice-standing- $900 \mathrm{MHz}$, under a NLOS scenario.

As can be seen, the log-normal distribution suitably models the power fluctuations in this case. Similar results have been obtained with tablet at $1940 \mathrm{MHz}$. The lognormal distribution describes the shadowing/masking effects in propagation.

Turning to the exposure investigation, by relying on the proportional relationship between the input power and the induced exposure, Figures 8 and 9 illustrate the cumulative distribution functions (CDF) of the $\mathrm{SAR}_{10 \mathrm{~g}}^{\text {peak }}$, induced by the input power in the posture voice-standing- $900 \mathrm{MHz}$ and in the case of posture data-standing-1940 $\mathrm{MHz}$, respectively.

With both postures, the $\mathrm{SAR}_{10 \mathrm{~g}}^{\text {peak }}$ is varied logarithmically over the ranges 0 and $1 \mathrm{~W} / \mathrm{kg}$.

A similar study was done for the $\mathrm{SAR}_{\mathrm{WB}}$. The parameters of obtained distributions are shown in Tables 2 and 3.

It is of practical interest to notice that, by setting $\alpha$ equal to $1 \mathrm{~mW}$, virtually all $S A R_{10 \mathrm{~g}}^{\text {peak }}$ and $\mathrm{SAR}_{\mathrm{WB}}$ values induced by the mobile phone and the tablet are less than thresholds required by ICNIRP [23]: $2 \mathrm{~W} / \mathrm{kg}$ for $\mathrm{SAR}_{10 \mathrm{~g}}^{\text {peak }}$ and $0.08 \mathrm{~W} / \mathrm{kg}$ for $\mathrm{SAR}_{\mathrm{WB}}$.
TABLE 3: Characteristic of the distribution of exposure values at $900 \mathrm{MHz}$.

\begin{tabular}{lccc}
\hline & $\mu(\mathrm{dB})$ & $\sigma$ & $\mathrm{CV}(\%)$ \\
& $\mu_{\mathrm{SAR}_{10 \mathrm{~g}}} / \mu_{\mathrm{SAR}_{\mathrm{WB}}}$ & & $\mathrm{CV}_{\mathrm{SAR}_{10 \mathrm{~g}} / \mathrm{CV}_{\mathrm{SAR}_{\mathrm{WB}}}}$ \\
\hline NLOS & $-3.18 /-8.00$ & 0.41 & $42 / 42$ \\
LOS+NLOS & $-2.95 /-7.77$ & 0.77 & $92 / 92$ \\
Only LOS & $-0.28 /-5.10$ & 0.92 & $112 / 112$ \\
\hline
\end{tabular}

TABLE 4: Characteristic of the distribution of exposure values at $1940 \mathrm{MHz}$.

\begin{tabular}{|c|c|c|c|}
\hline & $\begin{array}{c}\mu(\mathrm{dB}) \\
\mu_{\mathrm{SAR}_{10 \mathrm{~g}}} / \mu_{\mathrm{SAR}_{\mathrm{WB}}}\end{array}$ & $\sigma$ & $\begin{array}{c}\mathrm{CV}(\%) \\
\mathrm{CV}_{\mathrm{SAR}_{10 \mathrm{~g}} / \mathrm{CV}_{\mathrm{SAR}_{\mathrm{WB}}}}\end{array}$ \\
\hline NLOS & $-4.67 /-10.67$ & 0.32 & $36 / 36$ \\
\hline LOS+NLOS & $-4.45 /-10.45$ & 0.8 & $125 / 125$ \\
\hline LOS & $-4.32 /-10.3251$ & 1.03 & $178 / 178$ \\
\hline
\end{tabular}

To evaluate the impact of the propagation scenario on the variability of the input power, we focused on the assessment of the coefficient of variation $(\mathrm{CV})$, given as the ratio of the standard deviation to the mean multiplied by $100 \%$. As shown in Tables 3 and 4, this statistical indicator increases from one scenario to another in the following increasing order: NLOS, NLOS+NLOS, and LOS.

In a LOS scenario, the input power is strictly depending on the antenna gain in the LOS direction, which can vary as a function of the user's orientation with respect to the base station. In such scenario, the large fluctuations of the input power are explained by the nonuniformity of the antenna's gain.

In a pure NLOS scenario, the contribution of various paths, characterized by a significant diversity, reduces the impact of the radiation pattern nonuniformity. Briefly stated, a certain averaging of the antenna gain is established by the random combinations of NLOS path, which allows reducing accordingly the variability of the input power. In a LOS and NLOS mixture, this multipath adjustment remains valid, but it is limited by the Rice factor $K$. With a higher $K$ value, the case is similar to a pure LOS scenario.

$\mu(\log (\cdot))$ and $\sigma$ are the mean $(\mathrm{dB})$ and the standard deviation, respectively.

\section{Downlink Exposure: Simulations Results and Statistical Analysis}

The downlink exposure is firstly investigated using a Huygens's box excited by a single plane arriving from different directions. In fact, the first simulations are devoted to assess the exposure induced by the LOS scenario, by assuming that most of the power emitted by the base station arrived from a single dominant direction.

In such case, for a given user's posture, three parameters have been considered: the direction of arrival (both azimuth and elevation angles), the polarization, and the amplitude.

Due to the resource constraints and computing time limitations, a spatial discretization of the arrival directions 


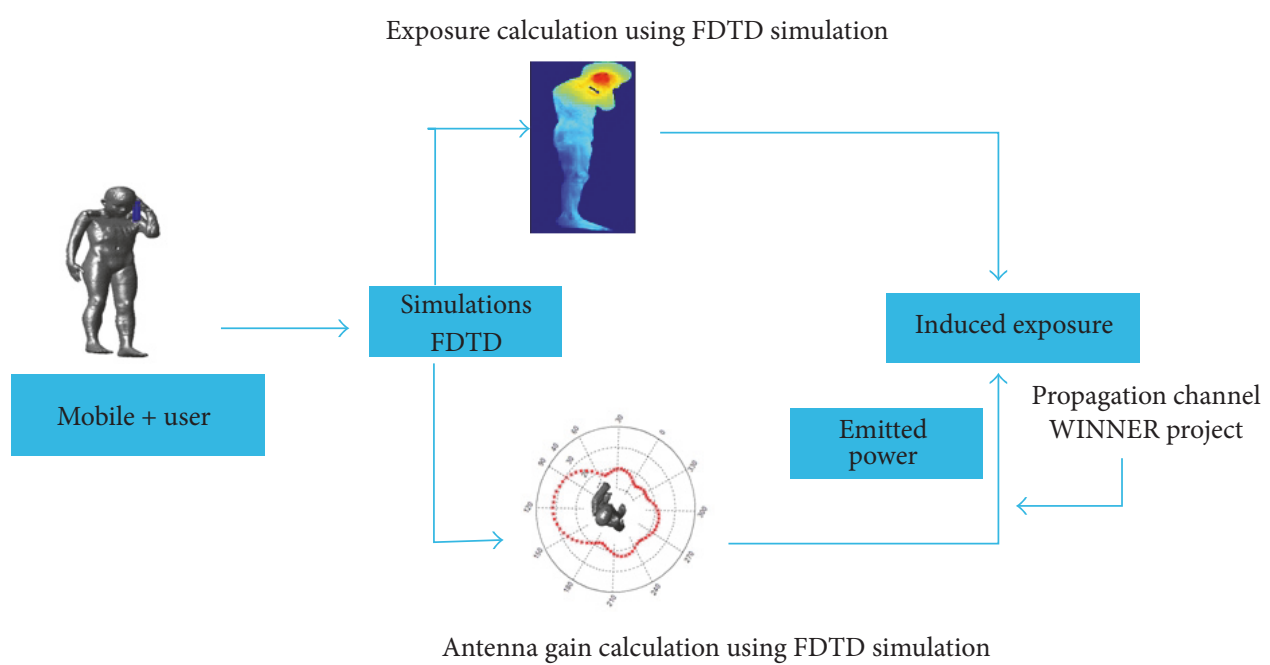

FIGURE 6: Flowchart of the subroutine for the characterization of the uplink exposure.

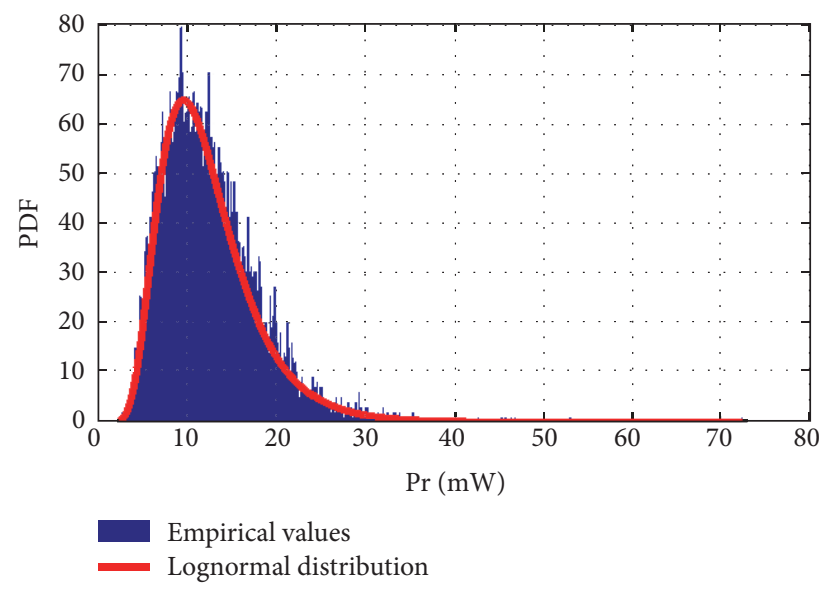

FIgURE 7: Comparison between empirical distribution of the input power delivered to the mobile phone during pure NLOS scenario and a log-normal distribution.

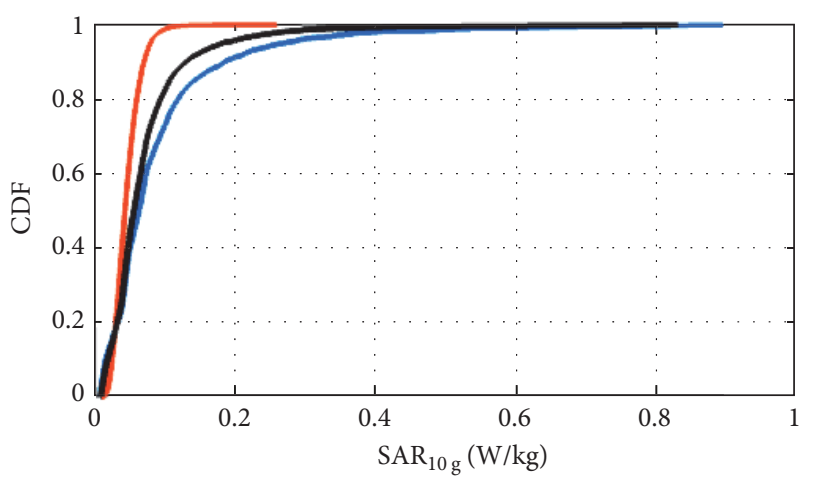

- Only LOS

— Indoor "in office": NLOS

— Urban "in street": LOS+NLOS

FIGURE 8: $\mathrm{SAR}_{10 \mathrm{~g}}$ induced by the mobile phone at $900 \mathrm{MHz}$.

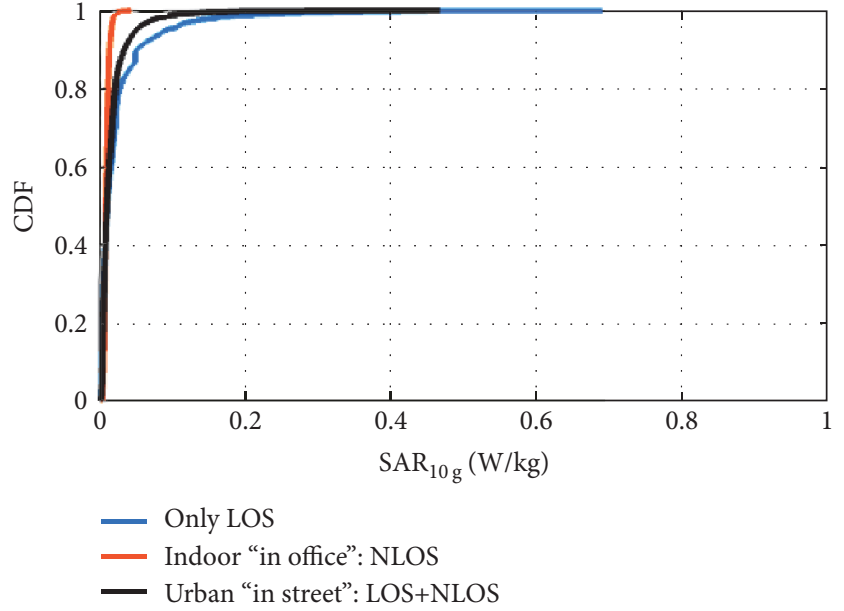

FIGURE 9: $\mathrm{SAR}_{10 \mathrm{~g}}$ induced by the tablet at $1940 \mathrm{MHz}$.

was refined, so as to reduce the number of simulations. Therefore, the azimuth angle $\phi$ has been discretized into 12 angles, uniformly spaced with a gap of 30 and the elevation angle $\theta$ ranged from -20 to 110 with a step width of 20 (3 angles). Both vertical (V) and horizontal $(\mathrm{H})$ polarization have been considered during each configuration, which increased the number of simulations to 72 for each posture. All parameters used in simulations are illustrated in Figure 10.

Figures 11 and 12 illustrate, respectively, the variation of $\mathrm{SAR}_{\mathrm{WB}}$ as a function of the elevation angle, azimuth angle, and the polarization for the postures voice-standing- $900 \mathrm{MHz}$ and data-standing-1940 $\mathrm{MHz}$. These results are obtained with an electric field of $1 \mathrm{~V} / \mathrm{m}$. In these figures, we can see that $\mathrm{SAR}_{\mathrm{WB}}$ is very sensitive to the azimuth angle $\phi$. Various curves of $\mathrm{SAR}_{\mathrm{WB}}$ show some sinusoidal behavior. As was mentioned in the research of Conil et al. [24], the power absorbed by the user's body is proportional to the exposed 


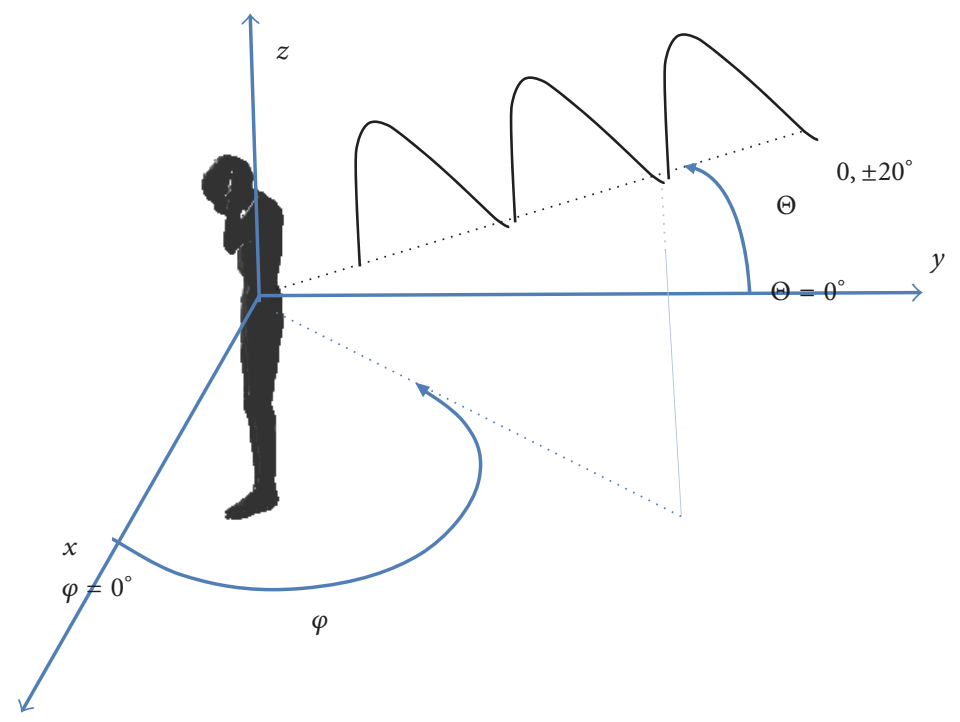

FIGURE 10: Definition of the parameters involved in the study of the exposure to a single plane wave.

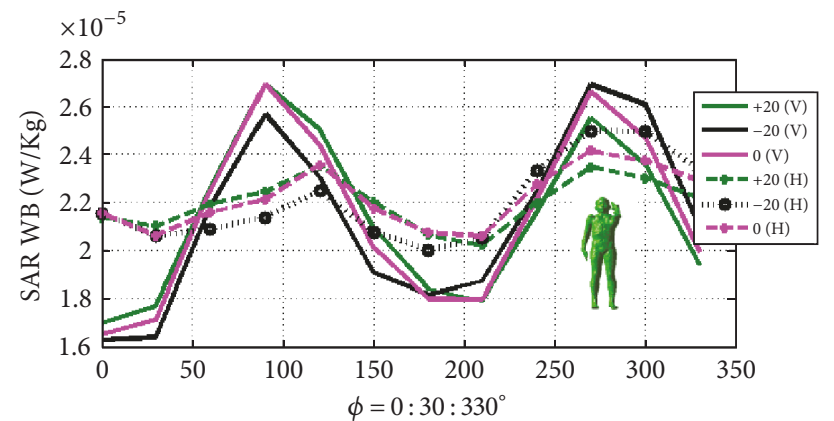

FIGURE 11: Variation of $\mathrm{SAR}_{\mathrm{WB}}$ obtained with the posture voicestanding-900 MHz after an exposure to a plane wave coming from three elevations, from azimuth from 0 to 360 and in both Vpolarization and $\mathrm{H}$-polarization.

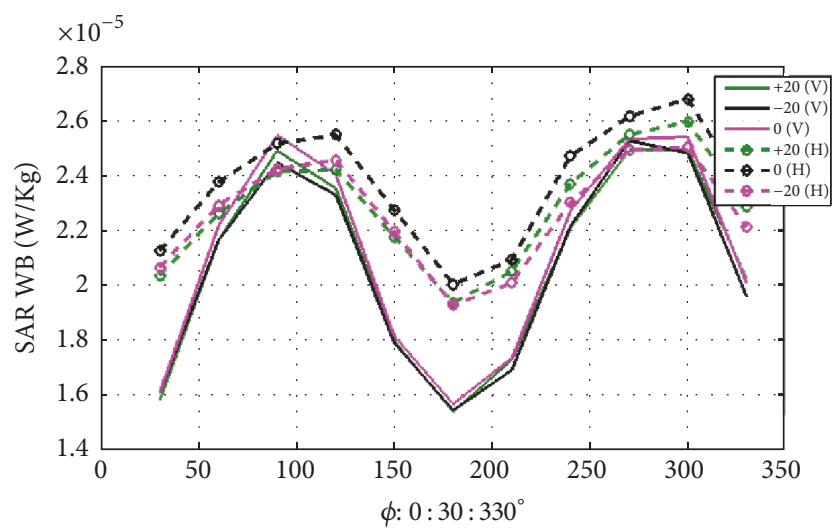

FIGURE 12: Variation of $\mathrm{SAR}_{\mathrm{WB}}$ obtained with the posture datastanding-1940 $\mathrm{MHz}$ after an exposure to a plane wave coming from three elevations, from azimuth from 0 to 360 and in both Vpolarization and H-polarization.

surface, at least for the frequencies here considered. This explains the origin of two peaks obtained at $\phi=270$ (frontal wave) and $\phi=90$ (back wave).

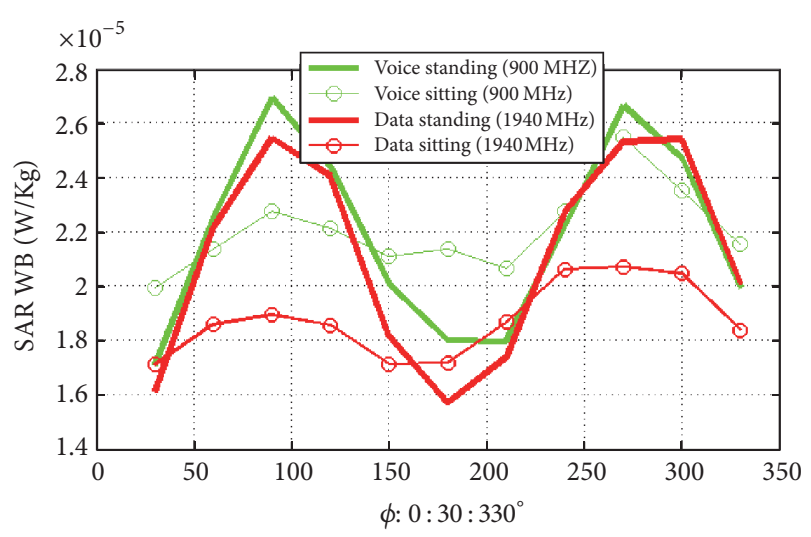

FIGURE 13: Comparison between the $\mathrm{SAR}_{\mathrm{WB}}$ induced in voice and data configurations (standing and sitting postures) at $900 \mathrm{MHz}$ and $1940 \mathrm{MHz}$, respectively. Results obtained after an exposure to plane wave vertically polarized at zero elevation.

The $\mathrm{SAR}_{\mathrm{WB}}$ also varies from one posture to another, which is basically due to the effective exposed surface of the body to the incoming wave. Figure 13 deals with a simple comparison between four specific postures: voice at (standing/sitting) and data (standing and sitting). The results show that, depending on the azimuth angle, the exposure obtained with a standing posture is almost always higher than that induced in a sitting posture. In fact, the $\mathrm{SAR}_{\mathrm{WB}}$ obtained with a sitting posture exceeds that induced in a standing posture only when the plane wave is coming from the left side of the phantom. This can be explained by the fact that a large part of the power is absorbed by the arms and the legs.

As regards the impact of polarization, for the frequency $900 \mathrm{MHz}$, the $\mathrm{SAR}_{\mathrm{WB}}$ induced by a plane wave vertically polarized (VP) is higher than that induced by horizontally polarized (HP) in the case of a back/frontal plane wave. Figure 14 illustrates the distribution of the power absorbed inside the child's body after an exposure to a frontal wave at 
$(\mathrm{V})$

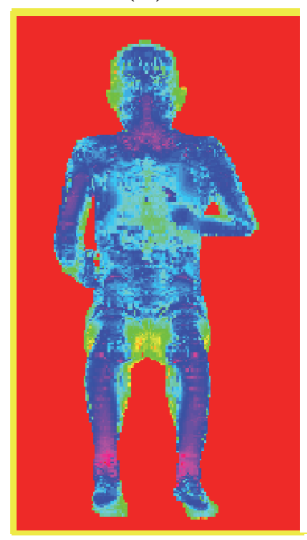

(H)

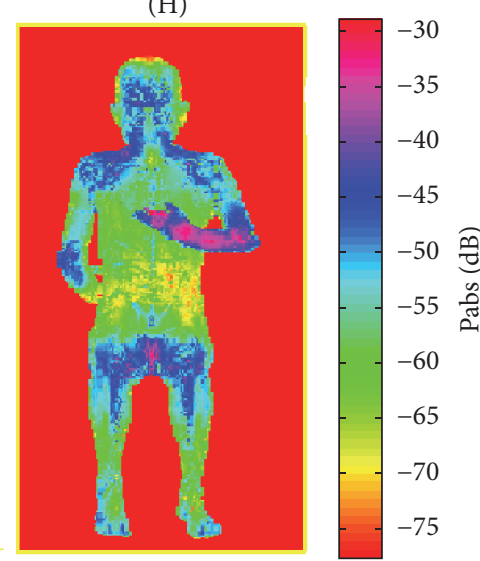

FIGURE 14: Distribution of the absorbed power inside the phantom after an exposure to a vertical/horizontal plane waves of $1 \mathrm{~V} / \mathrm{m}$.

$900 \mathrm{MHz}$ with both vertical and horizontal polarization. The exposure induced by HP becomes higher than that caused by $\mathrm{VP}$ on the side of phantom.

According to results given in $[25,26]$, this tendency must be true only with the frequencies below $1 \mathrm{GHz}[25,26]$.

In fact, for the frequency $900 \mathrm{MHz}$, the wavelengths $(0.33 \mathrm{~m})$ are, respectively, equal to the user's width and $1 / 4$ of its height. Hence, the user's body acts as a receiving antenna that favors wave absorption in both vertical and horizontal polarization. This is valid in, so far as the frequency is lower than $1 \mathrm{GHz}$, that when increasing the frequency, the exposure induced by horizontally polarized waves becomes higher than that induced by vertically polarized waves.

More clearly, for higher frequencies, the user's height begins larger than the wavelength (8 times higher at $1940 \mathrm{MHz}$ ), while the user's width remains relatively close to this value (twice higher at $1940 \mathrm{MHz}$ ). Consequently, the user's body becomes more sensitive to horizontally polarized waves. As shown in Figure 12, the $\mathrm{SAR}_{\mathrm{WB}}$ obtained at 1940 Mhz with horizontal polarization is higher than that obtained with vertical polarization.

Regarding the local exposure, we noticed that the $S A R_{10 \mathrm{~g}}^{\text {peak }}$ is sensitive to the dielectric properties of the tissues as well as the emitting frequency. However, the variation of $S_{A R} R_{10}^{\text {peak }}$ depending on the azimuth arrival angle does not exhibit a remarkable visual behavior.

Consequently, the efforts were concentrated only to identify the location of the peak value.

In this context, Figure 15 deals with the locations of various peaks in the postures voice-standing- $900 \mathrm{MHz}$, voicesitting-900 MHz, and data-sitting-1940 MHz. With both voice postures, the maximum is reached at the arms and feet, while with the data-sitting-1940 $\mathrm{MHz}$ the peak is located at the arms and the head. In fact, the exposure peaks depend basically on tissue types as well as the electromagnetic resonance. Particularly, at $900 \mathrm{MHz}$, this resonance occurs close to the ground. This may explain the presence of several peaks of exposure at the feet.
The motivation behind the peak identification is to predict if some accumulation of the local exposure in a given position can be expected, especially when the phantom is exposed to the plane wave and the wireless device together. Simultaneous uplink/downlink exposure is highly frequent in the context of wireless networks. In fact, it is important to anticipate if the peak induced by a multiple-exposure can be higher than that induced by each path separately. A graphic illustration of all local maximum allows evoking the possibility of accumulation of both peaks associated with both links in the same location. In such case, the local exposure may exceed the limit imposed by the ICNIRP. However, Figure 15 confirms that the probability of such superposition is always negligible.

5.1. Metamodeling Approach for Exposure Assessment. The single-wave exposure remains a singular case that does not reflect all of reality, which is often characterized by various multipath configurations. For that reason, we address in this section the exposure resulting from multiple plane waves having random propagation characteristics. In such scenario, the exposure assessment could be much costly in computation time and resources than that conducted with personal devices. In fact, contrary to the uplink case, where we were only interested in modeling the variability of the input power during the postprocessing of FDTD simulations, the evaluation of the downlink exposure requires performing a large number of simulations to examine all possible multipaths configurations. Even if a suitable discretization of the spatial domain can largely reduce the number of simulations $[27,28]$, this technical approach cannot be applied to some propagation scenarios, particularly in the presence of diffuse scattering components. Therefore, the numerical calculation process requires a very fine angular grid of arrival angles, which can increase dramatically the number of FDTD simulations.

To cope with this constraint, we propose to use a statistical approach through an input-output metamodel allowing approximating analytically the $\mathrm{SAR}_{\mathrm{WB}}$ induced by a given propagation scenario. The determination of this transfer function requires firstly performing an initial set of FDTD simulations, whose input parameters are generated using various probability distributions.

The obtained results are then used to extract an inputoutput function allowing to characterize all interactions suffered by input parameters as well as their relationship with the output quantity $\left(\mathrm{SAR}_{\mathrm{WB}}\right)$.

We rely on classical regression techniques to compute this transfer function which will be exploited to predict analytically the $S A R_{\mathrm{WB}}$.

Among the most popular methods used in such problems, we mention the Polynomial Chaos (PC) expansion, well known for its simplicity and efficiency.

In essence, this method involves determining the transfer function by expanding it over an orthogonal polynomial basis, such as the Legendre polynomials.

Its principle consists in determining a transfer function between the output $\left(\mathrm{SAR}_{\mathrm{WB}}\right)$ of the FDTD simulations and all 


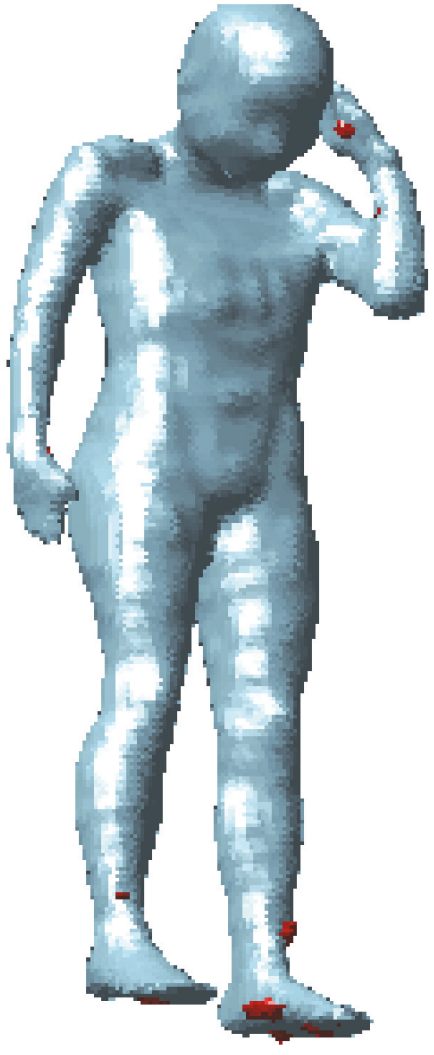

$400 \mathrm{MHz}$

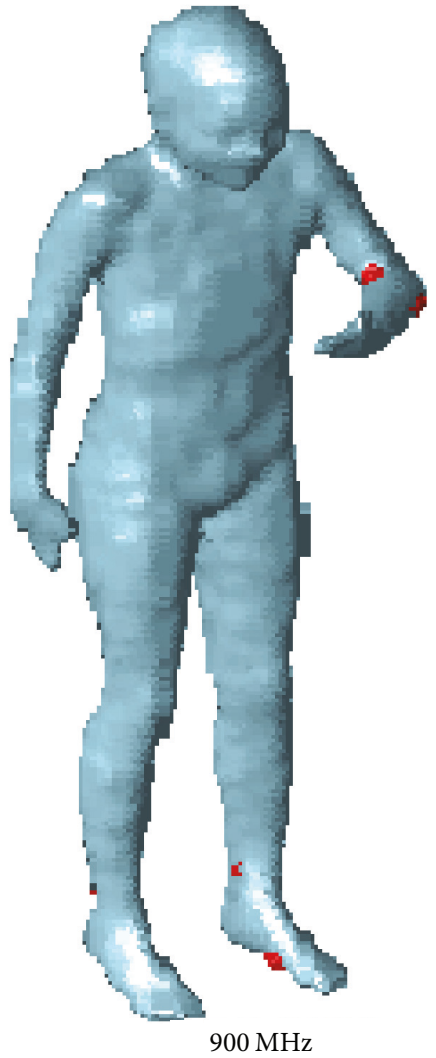

(b)

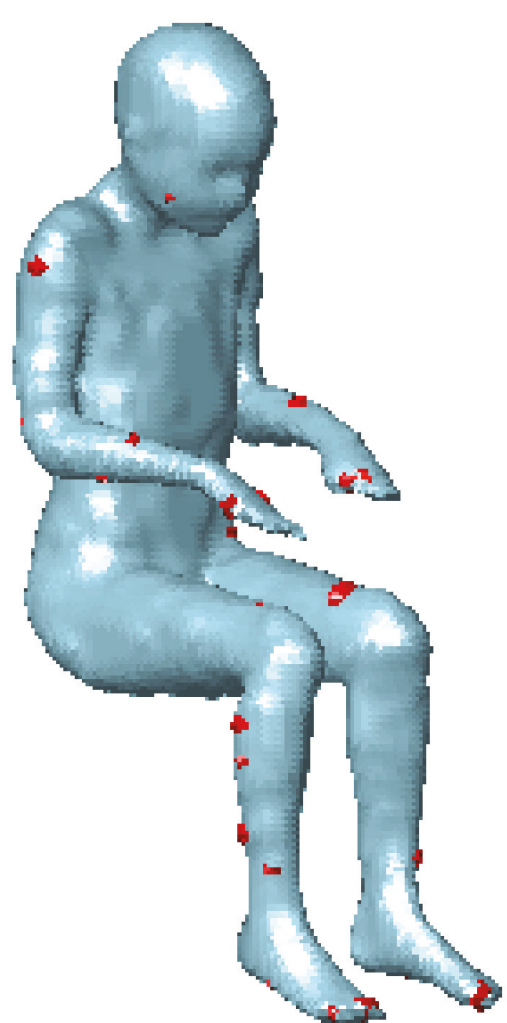

$1940 \mathrm{MHz}$

(a)

(a) Voice-standing-900 MHz, (b) voice-sitting-900 MHz, and (c) data-sitting-1940 MHz.

input parameters by expansion in an orthogonal polynomial basis, such as the Legendre polynomials.

Derived from this algebra approach, the obtained $\mathrm{SAR}_{\mathrm{WB}}$ should be written as

$$
\mathrm{SAR}_{\mathrm{WB}}^{\mathrm{PC}}=\sum_{k=1}^{M} \beta_{k} \Psi_{k}(X)
$$

where $\beta=\left\{\beta_{1}, \ldots, \beta_{M}\right\}$ are unknown determinist coefficients and $\Psi($.$) are the multivariate orthogonal polynomials,$ which are chosen to be the Legendre polynomials. $X=$ $\left\{X_{1}, \ldots, X_{N}\right\}$ is the input vector that regroups all parameters involved in the formulation of the ambient electric field (see (5)), including the amplitude $E_{n}$, the azimuth angle $\phi_{n}$, the elevation angle $\theta_{n}$, the phase $\alpha_{n}$, and the cross polarization ratio $\mathrm{XPR}_{n}$.

For ensuring a good spatial coverage of the experimental region with a finite number of samples, we relied on the Latin hypercube sampling (LHS) [29] to generate all parameters values. The various values provided by LHS are probabilities $p_{X_{i, i \in[1, N]}}$ that range from zero to one. Hence, to use these probability values in FDTD simulations, we need to calculate the realization associated with each probability using the inverse cumulative distribution function (ICDF) related to each propagation parameter.

In order to achieve the interorthogonality of the polynomials, especially when using the Legendre polynomials, it is necessary to translate the probabilities given by LHS into new variables varying between -1 and +1 . To ensure this constraint, we apply the linear transformation that associates a probability $p_{X_{i}}$ with the value $2 p_{X_{i}}-1$.

It is clear that the key step in this approach is the determination of weighting coefficients $\beta_{i}$. To do this, we use the regression approach to estimate the coefficients that minimize the Mean Squared Distance (MSD) between the analytic approximation $\mathrm{SAR}_{\mathrm{WB}}^{\mathrm{PC}}$ and the empirical value $\mathrm{SAR}_{\mathrm{WB}}^{\mathrm{FDTD}}$.

$$
\left\{\beta_{i, i \in[1, M]}\right\}=\arg \min \{A\}
$$

where

$$
A=\left\{\sum_{j=1}^{N_{\text {sim }}}\left\|\operatorname{SAR}_{\mathrm{WB}}^{\mathrm{FDTD}}\left(X^{(j)}\right)-\operatorname{SAR}_{\mathrm{WB}}^{\mathrm{PC}}\left(X^{(j)}\right)\right\|^{2}\right\},
$$

where $N_{\text {sim }}$ and $X^{(j)}$ are, respectively, the total number of simulations and the input vector used in the $j$ th simulation. 


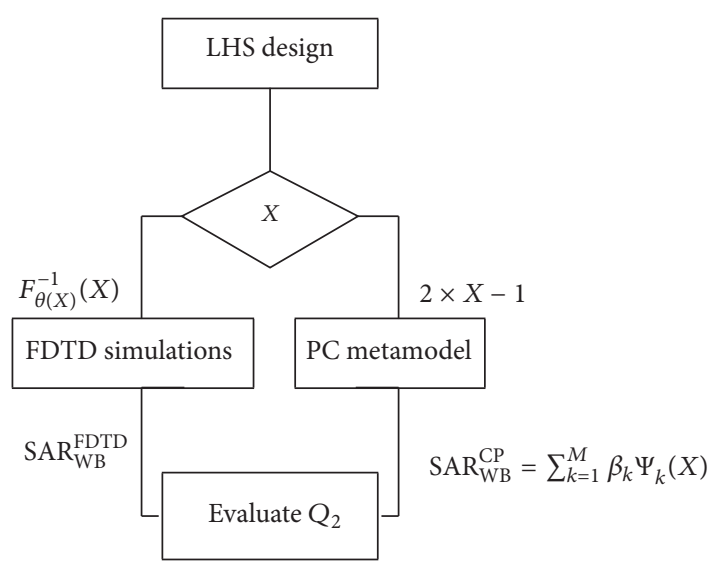

FIGURE 16: Flowchart of the proposed approach.

To judge the fidelity and the performance of the final metamodel, we conducted a certain number of validation tests by relying on the leave-one-out (LOO) approach [30]. This technique consists of removing one simulation from the total number of simulations and approximating a new surrogate model using only $N_{\text {sim }}-1$ simulations. The novel surrogate model should be used then to reestimate analytically the output of the removed simulation as well as the induced error with respect to the empirical result (given by the FDTD computation).

The average error $\epsilon$ is given as follows:

$$
\epsilon=\frac{1}{N_{\text {sim }}} \sum_{j=1}^{N_{\text {sim }}}\left\|\operatorname{SAR}_{\mathrm{WB}}^{\mathrm{FDTD}}\left(X^{(j)}\right)-\operatorname{SAR}_{\mathrm{WB}}^{\mathrm{PC}, j}\left(X^{(j)}\right)\right\|^{2},
$$

where $\mathrm{SAR}_{\mathrm{WB}}^{\mathrm{PC}, j}$ is the analytic model, obtained without considering the $j$ th simulation. In what follows, we use the error coefficient $Q^{2}$ as a quality criterion, which is calculated as follows:

$$
Q^{2}=1-\frac{\epsilon}{E\left\{\left\|\operatorname{SAR}_{\mathrm{WB}}^{\mathrm{FDTD}}\right\|\right\}} .
$$

A coefficient $Q^{2}$ of 1 indicates a perfect metamodeling. Figure 16 shows a flowchart illustrating the process of the exposure metamodeling.

To simplify the notation, the input vector $\mathbf{X}$ is arranged in the following order:

(i) $X_{1, j}$ is the amplitude $\left\|E_{n}^{(\mathrm{V})}\right\|$.

(ii) $X_{2, j}$ is the azimuth $\phi_{n}$.

(iii) $X_{3, j}$ is the elevation $\theta_{n}$.

(iv) $X_{4, j}$ is the phase $\alpha_{n}$.

(v) $X_{5, j}$ is the cross polarization $\mathrm{XPR}_{n}$.

It should be noted that the number of input parameters (size of $\mathbf{X}$ ) is some multiple $N_{p}$ (number of plane waves). To ensure a fast convergence of the regression algorithm, with a reasonable number of simulations, $N_{p}$ is assumed to be 5 , which remains a very reasonable choice [31].

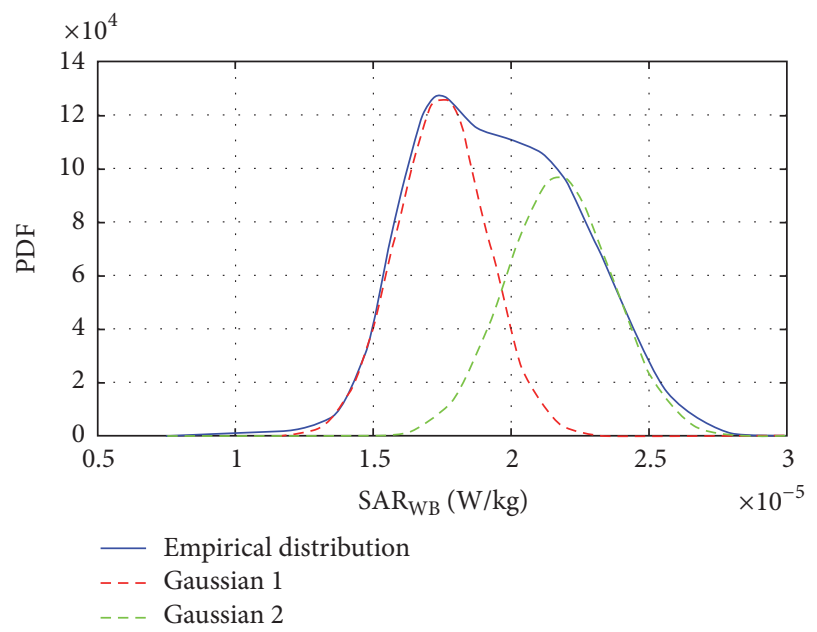

FIGURE 17: Fit of the $\mathrm{SAR}_{\mathrm{WB}}$ distribution with a Gaussian mixture.

Due to a limited time budget, we are interested only in the exposure induced in both models voice-standing- $900 \mathrm{MHz}$ and data-standing-1940 $\mathrm{MHz}$.

A set of 1000 FDTD simulations are performed with each model whose obtained results are discussed below.

Beginning with the voice-standing-900 MHz, Figure 17 shows the $\mathrm{SAR}_{\mathrm{WB}}$ distribution obtained from 1000 simulations. The drawn distribution resembles a Gaussian mixture (GM), which represents two significant "shoulders." On the other hand, the distribution width is too narrow, with a coefficient of variation of $14 \%$. Unlike the results obtained with a single plane wave, the $\mathrm{SAR}_{\mathrm{WB}}$ converged to an "average configuration" having poor sensitivity to the arrival directions of the electromagnetic waves. We also found that the exposure induced by 5 waves is often lower than that induced by a single frontal plane wave (considered as a worst case), when considering the same incident electric field $(1 \mathrm{~V} / \mathrm{m})$. The number of cases where the exposure exceeds the classical worst case is less than 5\% (see the colored region in Figure 18).

Turning to the metamodeling process, Figure 18 presents a graphical comparison between the empirical distribution $\mathrm{SAR}_{\mathrm{WB}}$ and that generated by the analytic metamodel when using the same input vector $\mathbf{X}$.

As regards the metamodeling fidelity, it was needed to increase the degree and the number of Legendre polynomials up 15 to obtain a $Q_{2}$ of 0.86 . This can be explained by the higher-order interactions existing between various input parameters.

The final step in the exposure metamodeling process is the prediction of the $\mathrm{SAR}_{\mathrm{WB}}$ for other multiple-waves realizations having similar propagation characteristics.

Figure 19 illustrates the distribution obtained from $10^{5}$ random configurations. The resulting distribution does not display two bumps (shown previously), but it gets close to a Gaussian distribution with a mean of $2.10^{-5}(\mathrm{~W} / \mathrm{kg})$ and a standard deviation of $15 \%$. It should be noted, however, that the mean and standard deviation of this distribution are similar to the results obtained with the empirical distribution. 


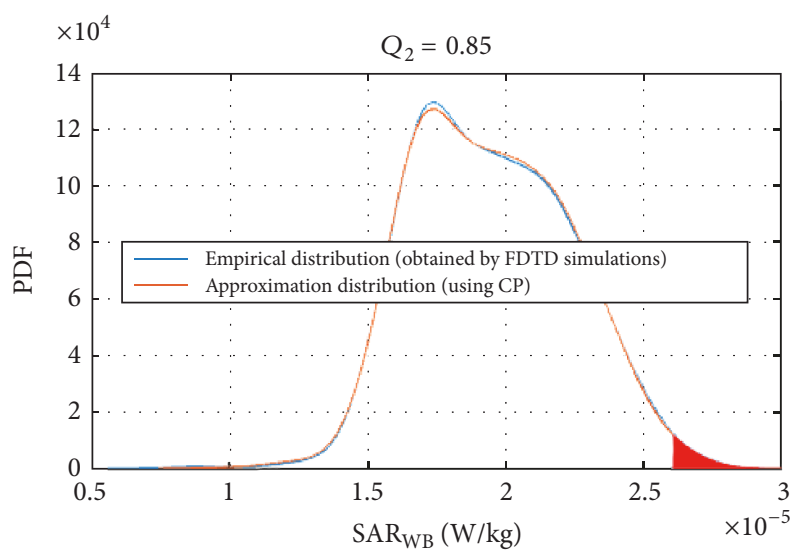

FIGURE 18: Comparison between the approximate and the empirical distribution.

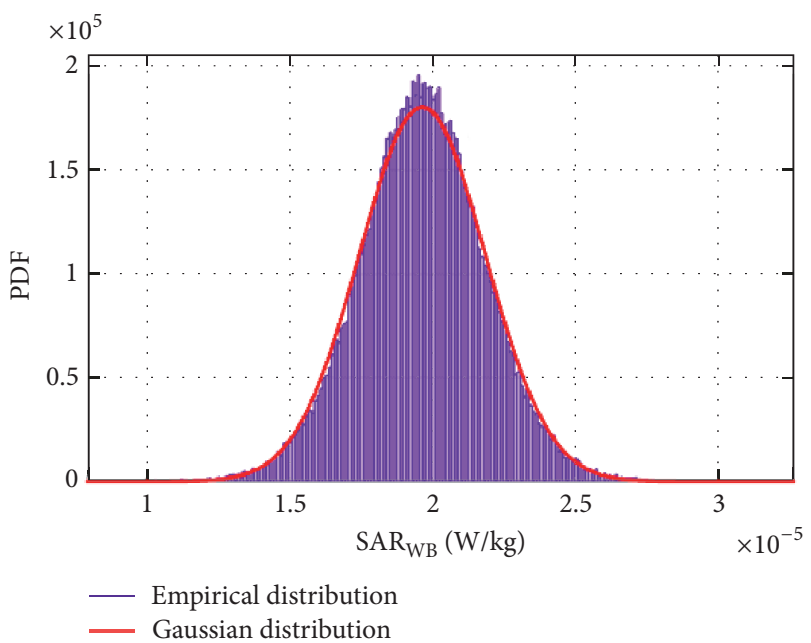

FIGURE 19: Distribution of the $\mathrm{SAR}_{\mathrm{WB}}$ induced by $10^{5}$ realizations.

This confirms the fact that the considered number of simulations, even if it makes to predict the "skeleton" of the transfer function (with a certain level of fidelity), remains insufficient to illustrate the final distribution as well as all interactions between 25 input parameters.

Similar results have been obtained with the datastanding-1940 MHz posture. An exposure metamodel derived from 1000 simulations is used to generate the $S A R_{W B}$ induced by $10^{5}$ random realizations. The obtained $\mathrm{CDF}$ is shown together with that calculated with data-standing-900 $\mathrm{MHz}$ in Figure 20.

Similar results are obtained with the posture datastanding-1940 MHz. An approximated metamodel of the exposure induced by random propagation scenarios was designed from 1000 FDTD simulations. This model was used to generate the SAR $\mathrm{WB}_{\mathrm{WB}}$ induced by $10^{5}$ different configurations. The obtained CDF is shown together with that calculated with voice-standing-900 $\mathrm{MHz}$ in Figure 20.

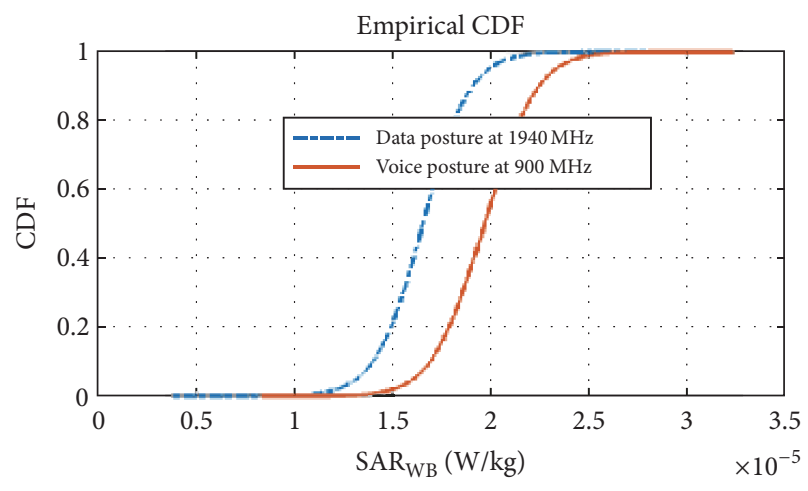

Figure 20: CDFs of the $\mathrm{SAR}_{\mathrm{WB}}$ obtained at $900 \mathrm{MHz}$ and $1940 \mathrm{MHz}$.

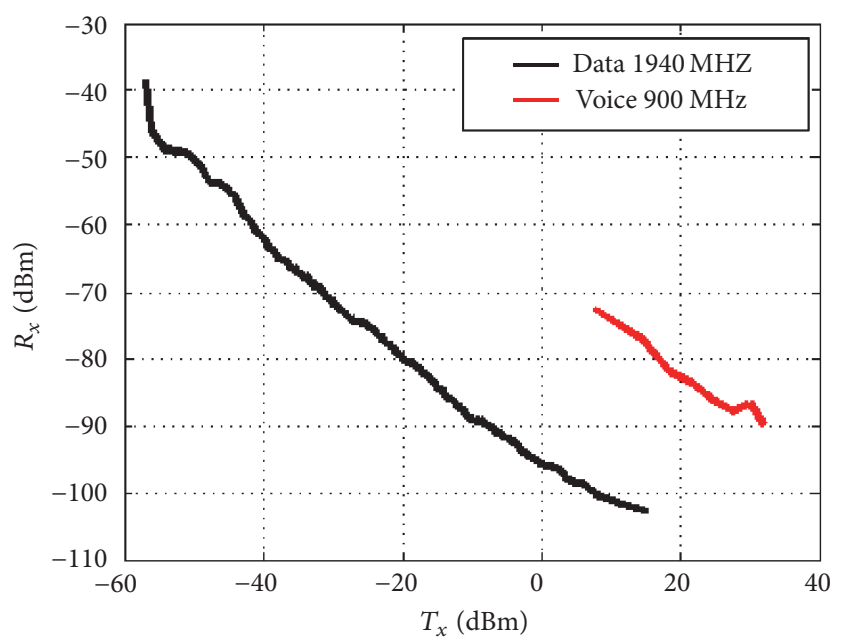

FIGURE 21: Variation of $R_{x}$ power as a function of $T_{x}$ power for $2 \mathrm{G}$ (900 MHz) and 3G systems (1940 MHz).

\section{Statistical Modeling of a Realistic Exposure Scenario}

This section is devoted to applying the methodologies presented as well as the obtained results to a real network. For this reason, various measurements of the uplink power $T_{x}$ (the power emitted by the user system) and the downlink power $R_{x}$ (the power received at the user system) are performed inside the Lyon city (France) and its surroundings cities, including basically NLOS scenarios. We note that the user system is the couple composed by the human body and the personal device. For emissions at $900 \mathrm{MHz}$, a hand-held probe connected to various base stations is configured to measure the radio traffic in both uplink and downlink paths. Concerning the tarfics at $1940 \mathrm{MHz}$, the data are taken from a measuring campaign conducted using two mobile phone trackers. A detailed description of measurement campaigns and an analysis of obtained results are given in [32].

As an example of results, Figure 21 illustrates the variation of the $R_{x}$ power as a function of the $T_{x}$ power. We can clearly see that the uplink power varies inversely as the downlink power, which can be explained by the use of advanced power management techniques in both GSM and 3G technologies. 


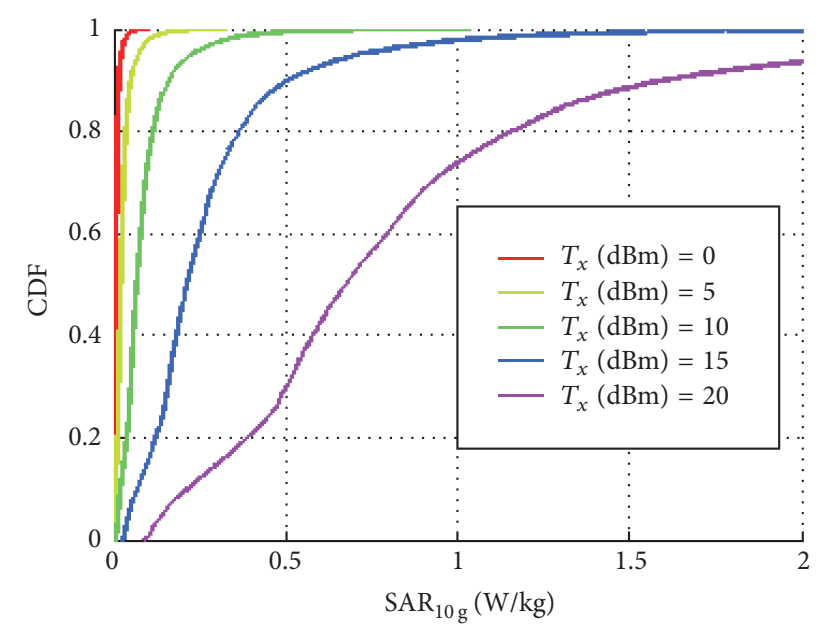

FIgURE 22: CDFs of $\mathrm{SAR}_{10 \mathrm{~g}}^{\text {peak }}$ induced by the mobile phone at $900 \mathrm{MHz}$ for various values of $T_{x}$.

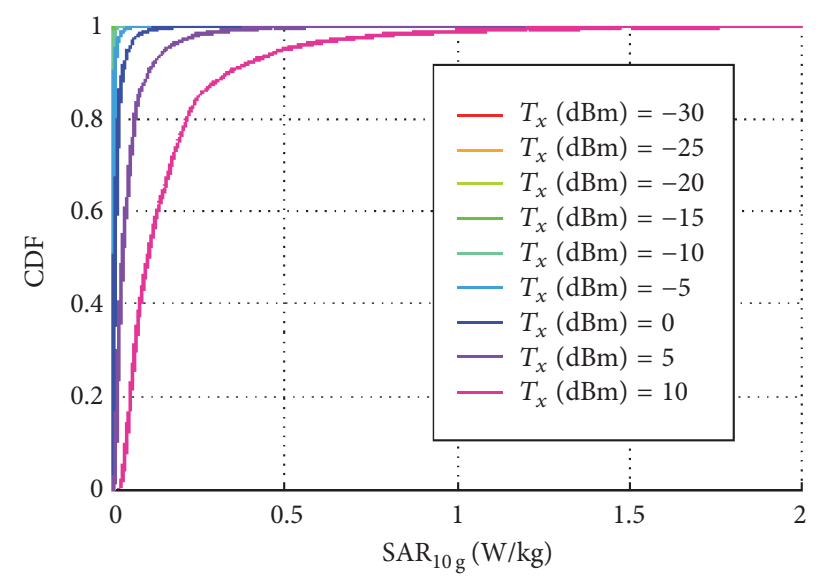

FIGURE 23: CDFs of $\mathrm{SAR}_{10 \mathrm{~g}}^{\text {peak }}$ induced by the tablet at $1940 \mathrm{MHz}$ for various values of $T_{x}$.

We recall that all $T_{x} / R_{x}$ values are harvested in the presence of the user's body, whether using a trace mobile or a probe connected to the network. In both cases, the measured power remains uncertain, which depends largely on the losses induced by the users body. Otherwise, the $T_{x}$ power can be considered as the given parameter $\alpha$ in (2) (Section 2). Hence, the exposure induced by varying $T_{x}$ values is not constant, but it follows a log-normal distribution, according to the fluctuations of the input power delivered to the mobile phone tracker. Therefore, the uplink exposure characterization process consists in extracting the exposure $\mathrm{CDF}$ for each $T_{x}$ power.

In what follows, we limit ourselves to the case of a pure NLOS scenario. As an example, Figures 22 and 23 show, respectively, the CDFs of the $\mathrm{SAR}_{10 \mathrm{~g}}^{\text {peak }}$ induced at $900 \mathrm{MHz}$ and $1940 \mathrm{MHZ}$. We note that the $\mathrm{SAR}_{10 \mathrm{~g}}^{\text {peak }}$ is generally lower than $2 \mathrm{~W} / \mathrm{kg}$. In fact, in the case of the mobile phone, the timeaveraged input power must not exceed $250 \mathrm{~mW}$ (for technical considerations). Consequently, the maximum of the resulting

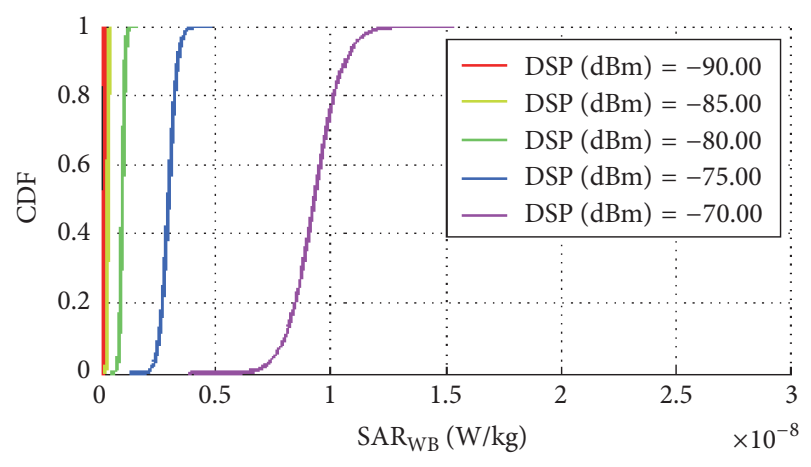

FIGURE 24: CDFs of $S_{\text {AB }}$ induced by the downlink (5 plane waves) at $900 \mathrm{MHz}$ for various values of DSP.

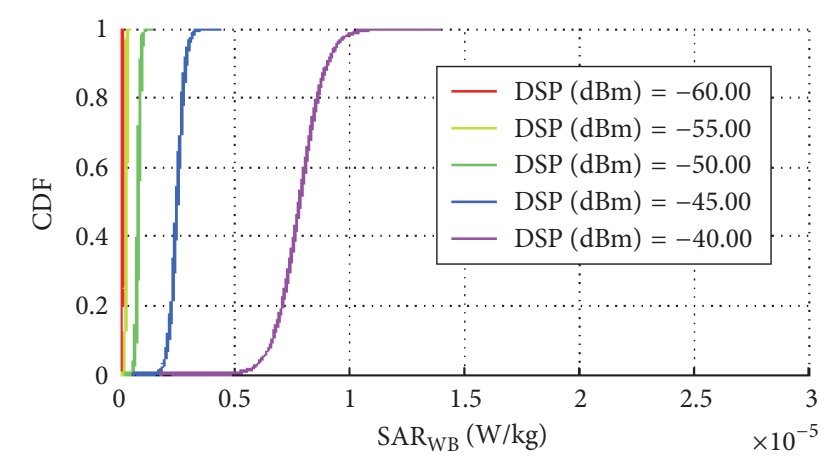

FIGURE 25: CDFs of $S_{A B}$ induced by the downlink (5 plane waves) at $1940 \mathrm{MHz}$ for various values of DSP.

$\mathrm{SAR}_{10 \mathrm{~g}}^{\text {peak }}$ remains below $0.9 \mathrm{~W} / \mathrm{kg}$. Similar results are obtained for the $S A R_{W B}$.

Turning to the downlink case, we draw attention to the fact that the power density DSP $\left(\mathrm{W} / \mathrm{m}^{2}\right)$ and the square of the ambient electric field $E_{a}^{2}$ can be calculated from the received power $R_{x}$ using the following mathematical relations:

$$
\begin{aligned}
\mathrm{DSP} & =\frac{1}{C_{i}} 10^{\left(R_{x}-30\right) / 10}, \\
E_{a}^{2} & =\frac{377}{C_{i}} 10^{\left(R_{x}-30\right) / 10},
\end{aligned}
$$

where $C_{i}$ is the aperture of a lossless isotropic antenna, which is equal to $\lambda^{2} / 4 \pi$. In fact, we consider here that the received system (the user's body + device) is a perfectly matched isotropic antenna, without considering the gain of the personal device. The objective of such hypothesis is to simplify the assessment of the downlink exposure (farfield) without taking into account the mobile phone mode (active/sleep). The evaluation of this quantity consists in using the analytic metamodel associated with the multiwave exposure (Section 5). Indeed, the electric field received is assumed to be a superposition of 5 plane waves. The rest of the downlink exposure characterization process consists in deducing the $\mathrm{SAR}_{\mathrm{WB}} \mathrm{CDF}$ using the proportional relationship between the exposure dose and the electric field.

As a preliminary result, Figures 24 and 25 illustrate the CDFs of the SAR $\mathrm{WB}_{\mathrm{B}}$ obtained from various values of DSP 
for each of the following scenarios: voice at $900 \mathrm{MHz}$ and data at $1940 \mathrm{MHz}$. Both figures indicate that the $\mathrm{SAR}_{\mathrm{WB}}$ is not significant, whose majority of values are less than $10^{-8} \mathrm{~W} / \mathrm{kg}$ $(900 \mathrm{MHz})$ and $10^{-5} \mathrm{~W} / \mathrm{kg}(1940 \mathrm{MHz})$.

The last part of this paper deals with the assessment of the total exposure which is given as the sum of both the uplink and downlink exposure (system of (1)). Due to the fact that the variation coefficient of the exposure is insignificant in the case of NLOS scenario, the contribution of each link (personnel devices/plane waves) can be simplified to the average of its associated distribution.

As regards the exposure at $900 \mathrm{MHz}$, the results show that the total exposure induced at $900 \mathrm{MHz}$ is largely dominated by the mobile phone radiation. This is partly due to the fact that the measured power $R_{x}$ is relatively low.

On the other side, three exposure cases have been observed with the tablet at $1940 \mathrm{MHz}$. In fact, we show that the levels of the local/global exposure are largely due to the downlink radiation, particularly when the $T_{x}$ power is below $40 \mathrm{~dB}$. Near this value, the resulting exposure is shared equally between the two paths. By increasing the $T_{x}$ power, the contribution of the tablet becomes significant.

\section{Summary and Conclusions}

This paper addresses the evaluation of both global and local radio wave exposure induced by a wireless network, based on FDTD simulations, on a simplified model of the propagation and on measurements. Beyond the sole examination of a few exposure configurations, it highlights that suitable methodological approaches as well as statistical techniques allow characterizing the variability of exposure, in relation to the characteristics of the radio channel. In the case of an exposure to a personal device, the use of a statistical propagation channel combined with shadowing effects induced by the user proximity allowed us to characterize the variability of the input antenna power as well as the resulting exposure. The results obtained showed that both quantities are lognormally distributed, regardless of the propagation scenario. Concerning the exposure induced by a superposition of plane waves, the application of metamodeling to the exposure via the $\mathrm{CP}$ method allowed consolidating the validity of the obtained statistical distributions with a limited number of realizations, namely, of greedy FDTD simulations. Even though the total exposure is often dominated by either uplink or downlink, we found out that the local exposure requires taking care of both radiation sources, especially in cases for which the difference is not enormous. Future studies will focus on the analysis of the exposure induced by other anatomical models, including babies and a pregnant women, under various propagation scenarios (in car, at smart home, femtocell, and so on).

\section{Conflicts of Interest}

The authors declare that they have no conflicts of interest.

\section{References}

[1] P. Gajšek, P. Ravazzani, J. Wiart, J. Grellier, T. Samaras, and G. Thuróczy, "Electromagnetic field exposure assessment in Europe radiofrequency fields $(10 \mathrm{MHz}-6 \mathrm{GHz})$," Journal of Exposure Science and Environmental Epidemiology, vol. 25, pp. 37-44, 2013.

[2] M. A. Jensen, "Application of FDTD and RGFM to EM interaction with biological tissue: a comparison," in Proceedings of the IEEE Antennas and Propagation Society International Symposium, vol. 4, pp. 1972-1975, Atlanta, Ga, USA, 1998.

[3] S. Tabbane, Handbook of Mobile Radio Networks, Artech House Publishers, London, UK, 2000.

[4] W. A. Shittu, B. G. Bajoga, F. Anwar, and M. J. E. Salami, "Prediction of received signal power and propagation path loss in open/rural environments using modified free-space loss and Hata models," in Proceedings of the IEEE International RF and Microwave Conference (RFM '08), pp. 126-130, Kuala Lumpur, Malaysia, December 2008.

[5] A. Sibille, "Statistical modeling of the radio-electric properties of wireless terminals in their environment," IEEE Antennas and Propagation Magazine, vol. 54, no. 6, pp. 117-129, 2012.

[6] Z. Wang, X. Chen, and C. G. Parini, "Effects of the ground and the human body on the performance of a handset antenna," IEE Proceedings: Microwaves, Antennas and Propagation, vol. 151, no. 2, pp. 131-134, 2004.

[7] Z. Wang, X. Chen, and G. C. Parini, "Body channel characterisation using dual band button antennas," Antennas and Propagation, pp. 958-961, 2009.

[8] M. A. Stuchly, A. Kraszewski, and S. S. Stuchly, "Exposure of human models in the near and far field-a comparison," IEEE Transactions on Biomedical Engineering, vol. 32, no. 8, pp. 609616, 1985.

[9] O. Lauer, P. Frei, M.-C. Gosselin, W. Joseph, M. Röösli, and J. Fröhlich, "Combining near- and far-field exposure for an organspecific and whole-body RF-EMF proxy for epidemiological research: a reference case," Bioelectromagnetics, vol. 34, no. 5, pp. 366-374, 2013.

[10] EU FP7 LEXNET (low EMF exposure future networks) project, http://www.lexnet-project.eu/.

[11] G. Blatman and B. Sudret, "Adaptive sparse polynomial chaos expansion based on least angle regression," Journal of Computational Physics, vol. 230, no. 6, pp. 2345-2367, 2011.

[12] D. Plets, W. Joseph, K. Vanhecke et al., "Joint minimization of uplink and downlink whole-body exposure dose in indoor wireless networks," BioMed Research International, vol. 2015, Article ID 943415, 2015.

[13] M. Tesanovic, E. Conil, A. De Domenico et al., "The LEXNET project: wireless networks and EMF: paving the way for lowEMF networks of the future," IEEE Vehicular Technology Magazine, vol. 9, no. 2, pp. 20-28, 2014.

[14] J. Liu, D. Wang, J. Wang et al., "Uplink power control and interference coordination for heterogeneous network," in Proceedings of the IEEE 23rd International Symposium on Personal, Indoor and Mobile Radio Communications (PIMRC '12), pp. 519523, Sydney, Australia, September 2012.

[15] A. Sibille, "Taking into account variability in wireless communication devices performance," in Proceedings of the 1st URSI Atlantic Radio Science Conference (URSI AT-RASC '15), Las Palmas, Spain, May 2015. 
[16] P. Kysti, "WINNER II channel models," IST-4-027756 WINNER II Deliverable D1.1.2, V1.2.4.2, 2008, http:// projects.celtic-initiative.org/winner+/phase_2_model.html.

[17] A. Krayni, A. Hadjem, A. Sibille, C. Roblin, and J. Wiart, "A novel methodology to evaluate uplink exposure by personal devices in wireless networks," IEEE Transactions on Electromagnetic Compatibility, vol. 58, no. 3, pp. 896-906, 2016.

[18] A. Thielens, G. Vermeeren, W. Joseph, and L. Martens, "Stochastic method for determination of the organ-specific averaged SAR in realistic environments at $950 \mathrm{MHz}$," Bioelectromagnetics, vol. 34, no. 7, pp. 549-562, 2013.

[19] A. Christ, W. Kainz, E. G. Hahn et al., "The virtual family-development of surface-based anatomical models of two adults and two children for dosimetric simulations," Physics in Medicine and Biology, vol. 55, no. 2, pp. N23-N38, 2009.

[20] IMST GmbH, User and Reference Manual for the 3D EM Time Domain Simulator, Empire, 2004, http://www.empire.de/ pagell.html.

[21] C. Gabriel, "Compilation of the dielectric properties of body tissues at RF and microwave frequencies, Brooks Air Force Base," Tech. Rep. AL/OE-TR-1996-0037, Brooks Air Force Base, Brooks, Tex, USA, 1996.

[22] S. Benkler, N. Chavannes, and N. Kuster, "New powerful FDTD source based on huygens surface: highly complex EM simulations performed on an ordinary PC," in Proceedings of the 31th Annu. Meeting Bioelectromagn. Soc., vol. 2009, pp. 14-19, Davos, Switzerland.

[23] ICNIRP, "Guidelines for limiting exposure to time-varying electric, magnetic and electromagnetic fields (up to $200 \mathrm{GHz}$ )," Health Physics, vol. 74, no. 4, pp. 494-522, 1998.

[24] E. Conil, A. Hadjem, A. Gati, M.-F. Wong, and J. Wiart, "Influence of plane-wave incidence angle on whole body and local exposure at $2100 \mathrm{MHz}$, IEEE Transactions on Electromagnetic Compatibility, vol. 53, no. 1, pp. 48-52, 2011.

[25] R. P. Findlay and P. J. Dimbylow, "Effects of posture on FDTD calculations of specific absorption rate in a voxel model of the human body," Physics in Medicine and Biology, vol. 50, no. 16, pp. 3825-3835, 2005.

[26] A. Hirata, N. Ito, and O. Fujiwara, "Influence of electromagnetic polarization on the whole-body averaged SAR in children for plane-wave exposures," Physics in Medicine and Biology, vol. 54, no. 4, pp. N59-65, 2009.

[27] T. Kientega, E. Conil, A. Hadjem et al., "A surrogate model to assess the whole body SAR induced by multiple plane waves at $2.4 \mathrm{GHz}$," Annals of Telecommunications, vol. 66, no. 7, pp. 419$428,2011$.

[28] G. Vermeeren, W. Joseph, and L. Martens, "Statistical multipath exposure method for assessing the whole-body SAR in a heterogeneous human body model in a realistic environment," Bioelectromagnetics, vol. 34, no. 3, pp. 240-251, 2013.

[29] M. D. McKay, R. J. Beckman, and W. J. Conover, "A comparison of three methods for selecting values of input variables in the analysis of output from a computer code," Technometrics, vol. 21, no. 2, pp. 239-245, 1979.

[30] T. Kientega, E. Conil, A. Gati et al., "Analysis of the distribution of specific absorption rate induced by five plane waves with a fast and new method in visible human," in Proceedings of the 30th URSI General Assembly and Scientific Symposium (URSIGASS '11), Istanbul, Turkey, August 2011.
[31] P. Refaeilzadeh, L. Tang, and H. Liu, "Cross validation," in Encyclopedia of Database Systems, pp. 532-538, Springer, New York, NY, USA, 2009.

[32] A. Gati, E. Conil, M.-F. Wong, and J. Wiart, "Duality between uplink local and downlink whole-body exposures in operating networks," IEEE Transactions on Electromagnetic Compatibility, vol. 52, no. 4, pp. 829-836, 2010. 


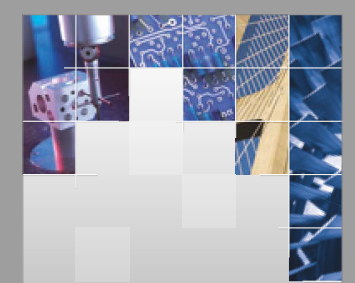

\section{Enfincering}
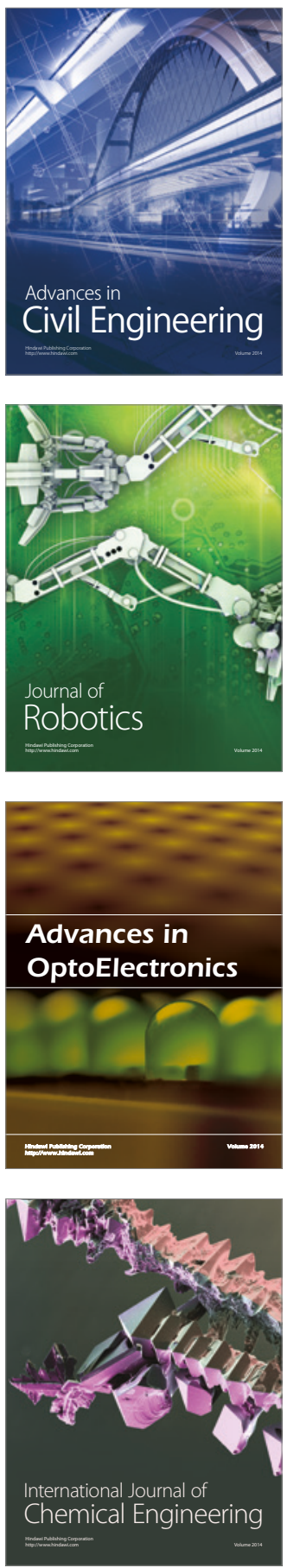

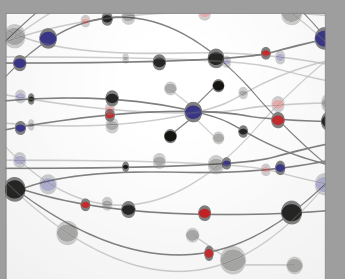

The Scientific World Journal

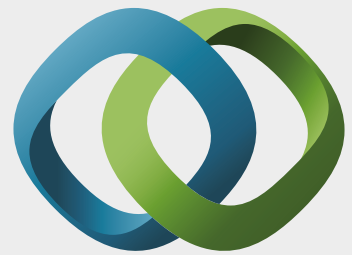

\section{Hindawi}

Submit your manuscripts at

https://www.hindawi.com
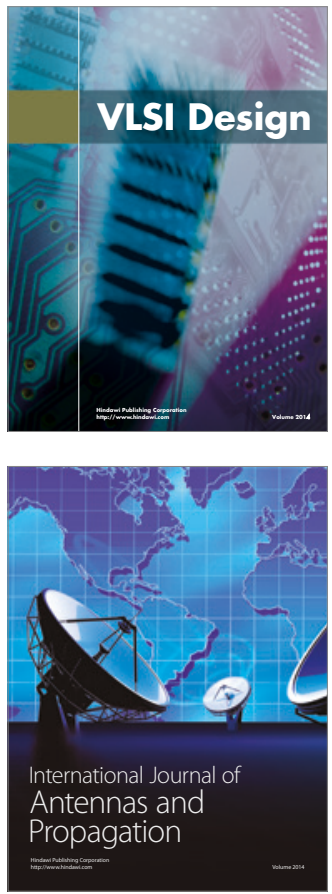

\section{Rotating}

Machinery
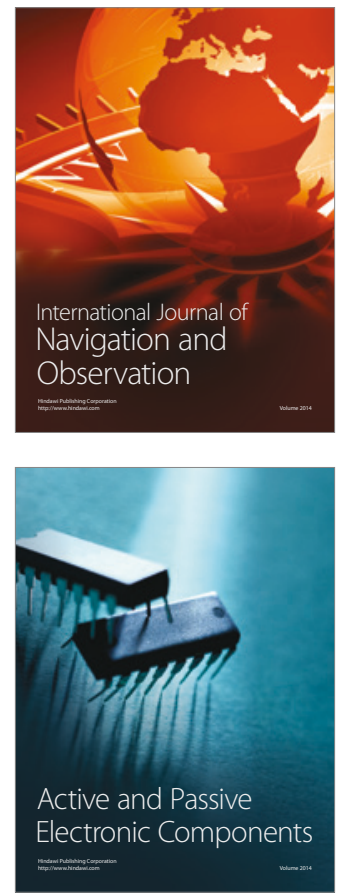
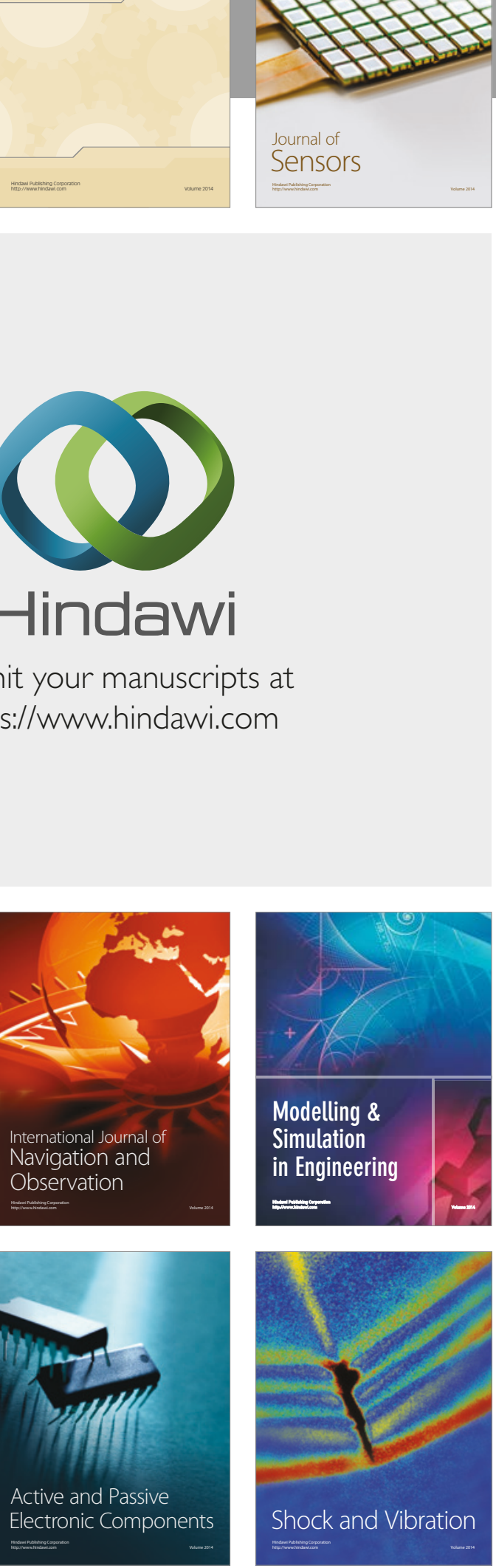
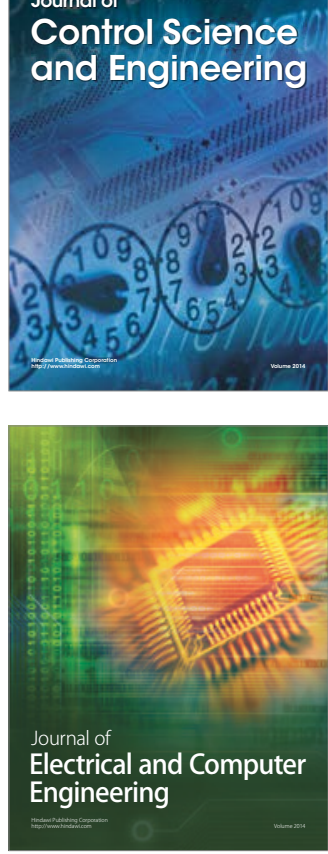

Distributed

Journal of

Control Science

and Engineering
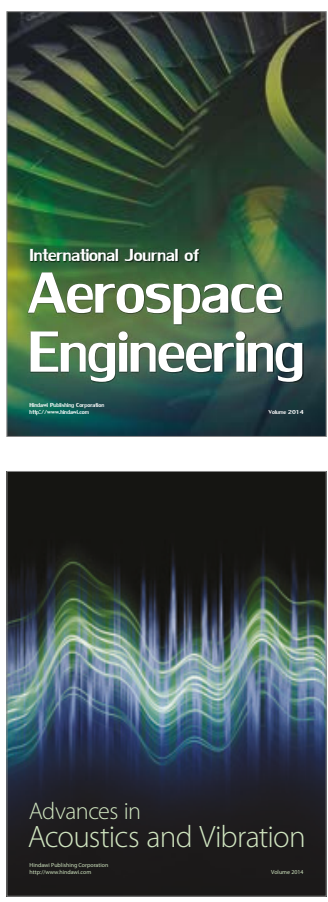

Sensor Networks 